\title{
Gas accretion from minor mergers in local spiral galaxies ${ }^{\star}$
}

\author{
E. M. Di Teodoro ${ }^{1}$ and F. Fraternali ${ }^{1,2}$ \\ 1 Department of Physics and Astronomy, University of Bologna, 6/2, Viale Berti Pichat, 40127 Bologna, Italy \\ e-mail: enrico.diteodoro@unibo.it \\ 2 Kapteyn Astronomical Institute, Postbus 800, 9700 AV Groningen, The Netherlands
}

Received 7 February 2014 / Accepted 28 May 2014

ABSTRACT

\begin{abstract}
We quantify the gas accretion rate from minor mergers onto star-forming galaxies in the local Universe using HI observations of 148 nearby spiral galaxies (WHISP sample). We developed a dedicated code that iteratively analyses HI data-cubes, finds dwarf gas-rich satellites around larger galaxies, and estimates an upper limit to the gas accretion rate. We found that $22 \%$ of the galaxies have at least one detected dwarf companion. We made the very stringent assumption that all satellites are going to merge in the shortest possible time, transferring all their gas to the main galaxies. This leads to an estimate of the maximum gas accretion rate of $0.28 M_{\odot} \mathrm{yr}^{-1}$, about five times lower than the average star formation rate of the sample. Given the assumptions, our accretion rate is clearly an overestimate. Our result strongly suggests that minor mergers do not play a significant role in the total gas accretion budget in local galaxies.
\end{abstract}

Key words. galaxies: interactions - galaxies: evolution - galaxies: kinematics and dynamics - galaxies: star formation galaxies: dwarf

\section{Introduction}

The evolution of galaxies is strongly affected by their capability of retaining their gas and accreting fresh material from the surrounding environment. Galaxies belonging to the so-called blue-sequence, which are actively forming stars and are dominated by young stellar populations, show an almost constant or a slowly declining star formation rate (SFR) throughout the Hubble time (e.g., Panter et al. 2007). Since the gas consumption time-scales are always of the order of a few Gyr (Noeske et al. 2007; Bigiel et al. 2011), spiral galaxies need to replenish their gas at rates similar to their star formation rates (Hopkins et al. 2008; Fraternali \& Tomassetti 2012). These arguments are fully applicable to the Milky Way: with an SFR of $1-3 M_{\odot}$ that slowly declined during the last 10 Gyr (e.g., Aumer \& Binney 2009; Chomiuk \& Povich 2011), the Galaxy would have exhausted its gas reservoir in a few Gyr without replacements from outside (e.g., Chiappini et al. 1997).

There are essentially two sources from which disc galaxies can gain new gas: the intergalactic medium (IGM) and other gas-rich galaxies. The IGM is the place where most baryons are thought to reside yet (e.g., Bregman 2007). Most of this gas is expected to be in a diffuse warm-hot phase (e.g., Shull et al. 2012). The IGM represents a huge reservoir of nearly pristine gas, but how this material can cool and accrete onto the discs is only poorly understood. Current cosmological simulations predict that gas accretion can occur in two modes (e.g., Ocvirk et al. 2008; Kereš et al. 2009): hot accretion, which dominates the growth of massive galaxies, and cold accretion through filamentary streams and clouds, which prevails in lower mass structures and at high redshifts (e.g., Dekel \& Birnboim 2006).

The second channel for gas accretion is through merger events. According to the extended Press-Schechter theory, the

\footnotetext{
* Appendix $\mathrm{A}$ is available in electronic form at http://www . aanda.org
}

structures in the Universe grow by several inflowing events and have increased their mass content through a small number of major mergers, more common at high redshifts, and through an almost continuous infall of dwarf galaxies (Bond et al. 1991; Lacey \& Cole 1993). Although several theoretical (e.g., Stewart et al. 2009; Kazantzidis et al. 2009) and observational studies (e.g., Patton et al. 2000; Lotz et al. 2008; Lambas et al. 2012) have been carried out in the past years, predictions and estimates for the galaxy merger rate and its evolution with redshift remain uncertain and no consensus has been achieved yet (e.g., Bertone \& Conselice 2009; Hopkins et al. 2010).

In this paper, we use neutral hydrogen (HI) observations to investigate gas accretion from minor mergers onto star-forming galaxies in the local Universe. The advantage of using HI observations instead of the optical-UV ones is that both morphological and kinematical information are immediately available. In addition, the gas layers are more easily disturbed by tidal interactions than the stellar disc. Two recent studies by Holwerda et al. (2011) and Sancisi et al. (2008) have taken advantage of HI data and used the WHISP catalogue (van der Hulst et al. 2001). Holwerda et al. (2011) focused on the galaxy merger fraction and, employing techniques developed for optical-UV observations, found a merger fraction of between $7 \%$ and $13 \%$. Instead, Sancisi et al. (2008) attempted to quantify the contribution of minor mergers to the total gas accretion. They found that $25 \%$ of the local galaxies show signs of minor interactions or have disturbed HI distribution and, assuming lifetimes for these observed features of about 1 Gyr and a typical accreted HI mass of the order of $10^{8}-10^{9} M_{\odot}$, they calculated an accretion rate of about $0.1-0.2 M_{\odot} \mathrm{yr}^{-1}$. This value is about an order of magnitude lower than typical star formation rates.

In this study, we use a quantitative approach to obtain a reliable estimate for the merger fraction and the gas accretion rate. We make use of a specific-purpose numerical code that is able to quickly analyse a large number of HI data-cubes, find dwarf 
gas-rich companions around disc galaxies, and estimate an upper limit for the gas accretion onto the discs. In Sect. 2, we describe the main features of our code and the methods we adopted. In Sect. 3, we show the results obtained by applying our analysis on a sub-sample of the WHISP catalogue, and we discuss them in Sect. 4.

\section{Method}

We wrote a numerical code to automatically identify 3D sources in data-cubes, that is, in image arrays with two spatial dimensions, related to the position on the plane of the sky, and one spectral dimension, which can correspond either to velocity or to frequency. Our code is targeted to work with HI observations as it performs a 3D scanning of the data to search for dwarf gasrich companions around large galaxies. When the program has found a candidate, it derives its physical properties, such as the HI mass, the projected distance from the main galaxy, and an estimate of the accretion rate onto the central disc. In short, the code used in this work is essentially a source finder plus an algorithm for estimating the accretion rate.

The standard flow of our code can be outlined in three steps:

1. Identifying the main galaxy. The pixels that belong to the central galaxy emission are identified and isolated through an appropriate mask.

2. Searching for dwarf galaxies. The data-cube is scanned for 3D sources, and dwarf galaxies or HI clouds inside the field of view are identified.

3. Estimating the gas accretion rate. For each detected dwarf, a maximum accretion rate onto the disc is calculated by estimating a minimum time of collision between the satellite and the central galaxy.

In the following sections, we describe the most important steps and the main features and limitations of our code.

\subsection{Searching for sources and background statistics}

The searching algorithm is derived from Duchamp, a code dedicated to 3D source detection (Whiting 2012) that was developed for the Australian SKA Pathfinder (ASKAP). The basic idea behind this algorithm is to locate and connect groups of bright and contiguous pixels that lie above some flux threshold, without imposing any size or shape requirement on the detection. The search is performed using either a 2D raster-scanning algorithm (Lutz 1980) or a 1D research along each individual pixel spectrum. Three-dimensional sources (two spatial dimensions and one spectral) are then built up on the basis of adjacency or neighbourhood criteria both in the velocity and in the spatial domain. For a full description of the source-finding algorithm, we refer to the Duchamp main paper. The 1D technique is less computationally expensive, but it can bring spurious detections. In contrast, the Lutz algorithm is generally more reliable at the price of computational slowness. As discussed later in this section, we used the 1D method to identify the main galaxy emission and the 2D technique to detect companions.

The searching algorithm can be schematically summarized as follows:

1. Pixel detection. The data-cube is scanned using one of the above-mentioned techniques, and a list of all pixels with a flux higher than a given threshold is produced.
2. Merging objects. The detected pixels that are considered to lie close to each other based on spatial and spectral requirements are merged. Adjacent detections or detections lying within a user-defined range of pixels or channels are expected to belong to the same object. After this step, a list of $3 \mathrm{D}$ sources is produced.

3. Growing objects. The size of the detections is increased by adding pixels at the edges of the objects that are above some secondary threshold. This step guarantees a smooth transition between the source and the background.

4. Rejecting objects. Not all detected objects can be considered reliable. Sources that do not match some agreement criteria, such as a minimum number of contiguous pixels and channels, are discarded.

A crucial point of the searching algorithm is the determination of the flux threshold above which a pixel can be considered as a part of a source. To do this, the code needs to estimate the central value and width of the noise distribution in the data-cube. The former should be zero or very close to zero for HI data-cubes without systematics (due for instance to problems with the data reduction). The typical data-cube of the WHISP survey is dominated by a large number of noise pixels and a relatively small number of bright pixels that belong to the sources. In this situation, it is preferable to calculate the noise over the whole datacube using the median as noise middle and the median absolute deviation from the median (MADFM) as noise spread. These quantities are less sensitive to the presence of very bright pixels than the equivalent normal statistics, the mean $\mu$ and the standard deviation $\sigma$. For a Gaussian distribution, the standard deviation can be written in terms of the MADFM as $\sigma=s / 0.6745$ (for details, see Whiting 2012, and references therein). The threshold $\tau$ is then determined with a simple sigma-clipping, that is, it is set at a number $n$ of standard deviations $\sigma$ above the median $m$ :

$\tau=m+n \sigma$.

This value is the minimum flux that a pixel must possess to be selected by the algorithm. We checked that the noise middle and spread calculated using the whole data-cube are the same as those obtained using only the channels with line emission; the differences do not exceed $5 \%$.

We used the searching algorithms in two different steps: the identification of the main galaxy, and the detection of satellites. The former consists of isolating all regions that belong to the main galaxy emission. The code performs a search in the datacube using the 1D technique and selects as the main galaxy the object that covers the largest number of pixels. This approach is not computationally expensive, and it is reliable when the code is analysing a heterogeneous group of galaxies, but it does not allow the code to identify systems in an advanced phase of merging, that is, when a companion is physically connected in space and velocity with the main galaxy. For the satellites, we used the Lutz algorithm, which guarantees a better reliability and minimizes the number of spurious detections. We imposed a neighbourhood criterion based on the spatial and spectral resolution of the observations: each detected pixel is merged with other detected pixels that lie within a spatial beam and within two velocity channels, which is the typical instrumental broadening full width at half maximum for HI observations when Hanning smoothing has been applied. Finally, we rejected all detections that are smaller than the beam area of the observations and less extended in velocity than the spectral broadening. We stress that a 3D source-finding algorithm, unlike the 2D methods, can isolate sources with different kinematics even if they are totally 
or partially overlapped in the plane of the sky. Indeed, if two sources have radial velocities that differ by more than the typical velocity resolution $\left(\sim 10-15 \mathrm{~km} \mathrm{~s}^{-1}\right)$, they are always detected as separate sources, no matter whether or not they overlap in the sky.

\subsection{Accretion and star formation rate estimates}

The main purpose of this study is to estimate the maximum gas accretion rate attributable to minor mergers. In the following we describe our assumptions.

First, we assumed that all dwarf galaxies will collide in the future with the main galaxies and that their gas will be entirely and instantaneously accreted. Second, we assumed that the collision will occur in the shortest possible time. To calculate this time, we caused the satellites to move in parabolic trajectories leading to impact the outer regions of the main galaxies. The orbit was defined in the $3 \mathrm{D}$ space by fixing the focus of the parabola at the centre of the main galaxy, imposing the passage through the satellite and fixing the position of the orbital pericentre at a distance equal to the maximum radius of the central galaxy (Fig. 1). For a generic conic orbit, the time-scale of collision can be obtained by using the equation of the true anomaly $v$ of celestial mechanics:

$\int_{0}^{v} \frac{\mathrm{d} v^{\prime}}{\left(1-e \cos v^{\prime}\right)^{2}}=\sqrt{\frac{\mu}{p^{3}}}\left(t-T_{0}\right)$,

where $e$ is the eccentricity of the orbit, $T_{0}$ is the time of the peri-centre passage, $p$ is the semi-latus rectum of the conic section, and $\mu=G\left(M_{\text {main }}+M_{\text {sat }}\right) \sim G M_{\text {main }}$ is the total dynamical mass of the system galaxy plus satellite multiplied by the gravitational constant $G$. The dynamical mass of the central galaxy $M_{\text {main }}\left(R_{\max }\right)=G^{-1} v_{\mathrm{c}}\left(R_{\max }\right)^{2} R_{\max }$ is calculated within the maximum radius $R_{\max }$ of the source, estimated by the searching algorithm. The circular velocity $v_{\mathrm{c}}$ is obtained from the velocity widths of the HI global profiles at the $20 \%$ of the peak flux corrected for the inclination taken from the HyperLEDA catalogue. By solving the integral (2) for parabolic orbits $(e=1)$, one obtains the following formula, which describes the variation of the true anomaly $v$ as a function of time:

$\tan \frac{v}{2}+\frac{1}{3} \tan ^{3} \frac{v}{2}=\sqrt{\frac{\mu}{p^{3}}}\left(t-T_{0}\right)$

where the semi-latus rectum for parabolic orbit is $p=2 R$, where $R$ is the distance between the focus and the vertex of the parabola (Fig. 1).

By using Eq. (3), we can estimate the time of the peri-centre passage $T_{0}$ by calculating $v$ through a de-projection of the projected anomaly $v_{\mathrm{p}}$ of the dwarf galaxy measured in the plane of the sky. The accretion rate of cold gas onto a certain galaxy is then obtained by dividing the HI mass of each dwarf by its time of peri-centre passage:

$\dot{M}_{\mathrm{HI}}=\sum_{i=0}^{n} M_{\mathrm{HI}, i} / T_{0, i}$,

where the sum is taken over all the detected companion galaxies. The HI mass $M_{\mathrm{HI}}$ is calculated from the flux density using the following relation (Roberts 1975):

$M_{\mathrm{HI}}=2.356 \times 10^{5} D^{2} \int S(v) \mathrm{d} v$,

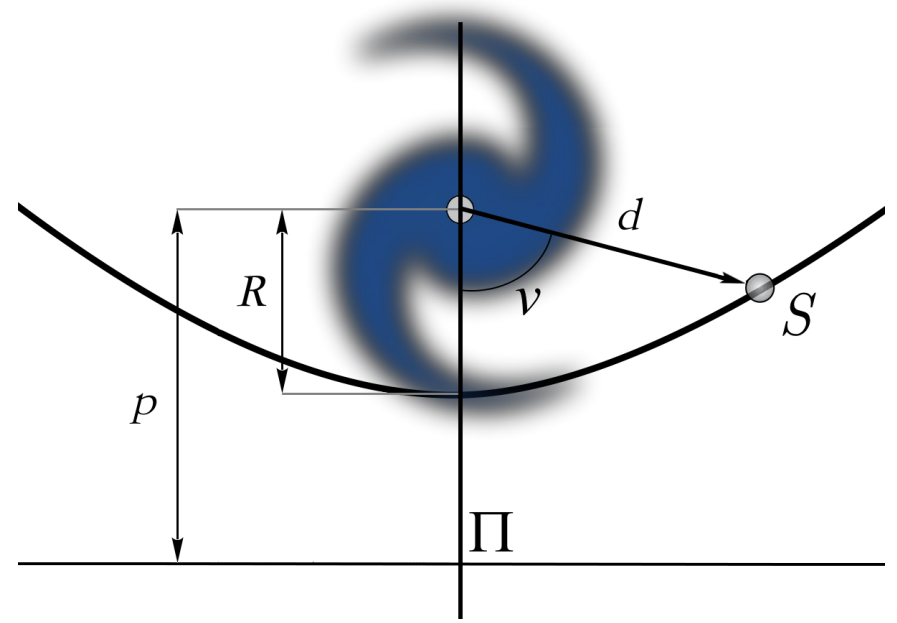

Fig. 1. Schematical view of the parabolic orbit approximation. The blue spiral is the main galaxy, $S$ is the satellite with projected distance $d$ and true anomaly $v$. The distance $p$ between the centre of the spiral galaxy and the directrix $\Pi$ of the parabola is twice the outer radius of the main galaxy $R$.

where $\int S(v) \mathrm{d} v$ is the integral across the line of the flux density corrected for the primary beam attenuation and expressed in $\mathrm{Jy} \mathrm{km} \mathrm{s}^{-1}$ and $D$ is the distance in Mpc. Equation (5) is valid under the assumption that the gas is optically thin, which is generally a good approximation for neutral hydrogen, especially in dwarf galaxies, therefore no correction for HI self-absorption was applied. The distances were preferably taken from the Extragalactic Distance Database (EDD, Tully et al. 2009), available for a number of galaxies with $v_{\text {sys }}$ up to $10000 \mathrm{~km} \mathrm{~s}^{-1}$ and mostly obtained from Cepheids, TRGB, SNIa, or the Cosmicflows-2 project (Tully et al. 2013). Otherwise, we used the NASA/IPAC Extragalactic Database (NED). For seven galaxies without available better estimates, we used the Hubble flow $D=v_{\text {sys }} / H_{0}$ with $H_{0}=70 \mathrm{~km} \mathrm{~s}^{-1} \mathrm{Mpc}^{-1}$ and the systemic velocity $v_{\text {sys }}$ corrected for Virgo-centric inflow using the values given by the HyperLEDA catalogue.

We compared the total gas accretion (4) with the gas depletion due to the star formation process in the discs. The SFR of the central galaxies was calculated from the far-infrared luminosities (Kennicutt 1998):

$S F R=\frac{L_{\mathrm{FIR}}}{2.2 \times 10^{43}} \quad M_{\odot} \mathrm{yr}^{-1}$,

with the $L_{\mathrm{FIR}}$ in $\mathrm{erg} \mathrm{s}^{-1}$ obtained from the far-infrared (FIR) flux defined after Helou et al. (1985) as

FIR $=1.26 \times 10^{-11}\left(2.58 f_{60 \mu}+f_{100 \mu}\right) \quad \mathrm{erg} \mathrm{s}^{-1} \mathrm{~cm}^{-2}$,

where $f_{60 \mu}$ and $f_{100 \mu}$ are the fluxes at 60 and 100 micron expressed in Jansky. In this work we used the IRAS fluxes taken from NED and HyperLEDA. All main galaxies in our sample are detected both at $60 \mu$ and $100 \mu$. See Table A.1 for their main physical properties.

\subsection{Major and minor mergers}

We split major and minor mergers depending on the baryonic mass ratio: pairs of galaxies with $M_{\text {bar,sat }} / M_{\text {bar,main }} \leq 0.20$ are classified as minor mergers, otherwise as major. We preferably estimated the baryonic mass as

$M_{\mathrm{bar}}=M_{*}+1.4 M_{\mathrm{HI}}$, 
where the factor 1.4 takes into account the helium gas fraction. We neglected the contribution of molecular gas. The HI mass $M_{\mathrm{HI}}$ is directly estimated from data-cubes through Eq. (5). A rough estimate of the stellar mass $M_{*}$ is obtained by using the total $K_{\mathrm{s}}$-band magnitude, corrected for extinction, taken from the 2MASS Redshift Survey (2MRS, Huchra et al. 2012) and adopting the following formula (e.g. Longhetti \& Saracco 2009):

$\log _{10}\left(M_{*}\right)=\log _{10}\left(M / L_{K}\right)-0.4\left[K+5-5 \log _{10}\left(D_{[\mathrm{pc}]}\right)-3.28\right]$

where $M / L_{K}$ is the stellar mass-to-light ratio (in solar units) in the $K$ band and 3.28 is the absolute $K$-band magnitude of the Sun in the Vega system (Binney \& Merrifield 1998). We assumed a constant value of mass-to-light ratio $M / L_{K}=0.6 M_{\odot} / L_{\odot, K}$, compatible with stellar population models (e.g. Portinari et al. 2004) with a Kroupa initial mass function (Kroupa 2002).

When 2MRS magnitudes were not available, which is the case for most dwarf satellites and a few main galaxies, we directly derived $M_{\text {bar }}$ from the baryonic Tully-Fisher relation (BTFR),

$\log _{10}\left(M_{\text {bar }}\right)=a \log _{10}\left(v_{\text {flat }}\right)+b$,

with $a=3.82 \pm 0.22$ and $b=2.01 \pm 0.41$ (McGaugh 2012). The $v_{\text {flat }}$ was assumed as half of the inclination-corrected velocity widths $w_{20}$ of the HI global profiles at the $20 \%$ of the peak flux. Since inclination angles are not known for most dwarf satellites, we adopted an average inclination of 60 degrees for these galaxies.

\subsection{Data sample}

The Westerbork HI survey of Irregular and Spiral galaxies Project (WHISP, van der Hulst et al. 2001) is a survey of the neutral hydrogen content in galaxies selected from the Uppsala General Catalogue (UGC, Nilson 1973) and is observed with the Westerbork Synthesis Radio Telescope (WSRT). To date, WHISP is the largest publicly available catalogue of HI nearby galaxies observed with an interferometer and includes galaxies at $\delta>20^{\circ}$ (B1950) with major axis apparent size $>1.2^{\prime}$ ( $B$ band) and HI flux densities $F_{\mathrm{HI}}>100$ mJy. Objects satisfying these selection criteria generally have systemic velocities lower than $6000 \mathrm{~km} \mathrm{~s}^{-1}$, that is, distances smaller than $85 \mathrm{Mpc}$ using the Hubble flow with $H_{0}=70 \mathrm{~km} \mathrm{~s}^{-1} \mathrm{Mpc}^{-1}$. The galaxies were chosen to be reasonably distributed over all Hubble types, even if later-type galaxies are favoured by the observational criteria. The highest spatial resolution for the WHISP data is $12^{\prime \prime} \times 12^{\prime \prime} / \sin (\delta)$, the typical channel separation is of the order of $5 \mathrm{~km} \mathrm{~s}^{-1}$. In this work, we used HI data-cubes spatially smoothed to $30^{\prime \prime} \times 30^{\prime \prime}$ and to $60^{\prime \prime} \times 60^{\prime \prime}$. The original sample comprises 256 data-cubes containing about 370 galaxies $^{1}$.

Since our goal is to study dwarf satellites around large starforming galaxies, we selected a sub-sample of spiral galaxies by keeping only the data-cubes that contained at least one galaxy with a rotation velocity $v_{\text {flat }}=w_{20} /(2 \sin i)>100 \mathrm{~km} \mathrm{~s}^{-1}$. The selection was performed through a cross-correlation between the $w_{20}$ estimated directly from the data-cubes and the $w_{20}$ calculated using the Tully-Fisher relation from Sakai et al. (2000),

$M_{B}=-(7.97 \pm 0.72)\left(\log w_{20}-2.5\right)-(19.80 \pm 0.11)$,

1 The datacubes, the column density maps, and the velocity fields of the WHISP galaxies, at $12^{\prime \prime}, 30^{\prime \prime}$ and $60^{\prime \prime}$ of resolution, are publicly available for the "Westerbork on the Web" project at ASTRON (http: //www . astron.nl/wow/).

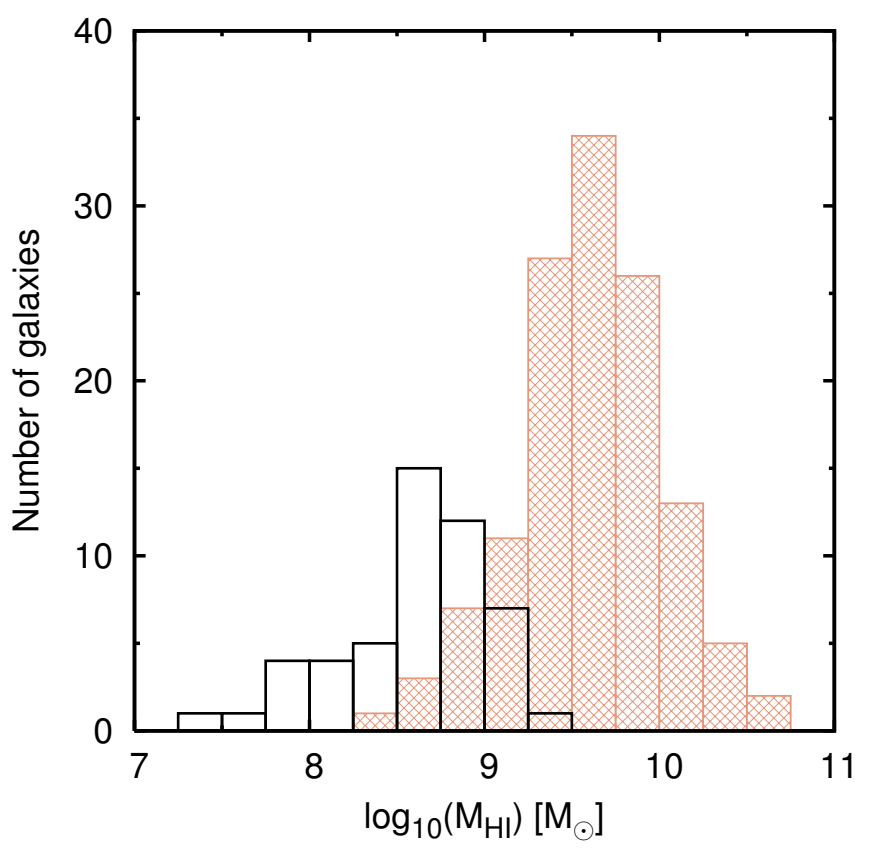

Fig. 2. HI mass distribution of the detected galaxies in the WHISP sample. Orange shadowed boxes show the spiral galaxies selected as $v_{\text {flat }}>100 \mathrm{~km} \mathrm{~s}^{-1}$, black boxes show their dwarf satellites.

where $M_{B}$ is the $B$-band absolute magnitude (corrected for galactic extinction and $k$-correction), taken from HyperLEDA. We kept only galaxies for which both methods returned $v_{\text {flat }}>$ $100 \mathrm{~km} \mathrm{~s}^{-1}$. This cross-correlation is needed to avoid spurious selections related to some unreliable inclination angles in the HyperLEDA catalogue. Our final sample has 148 data-cubes. Spiral galaxies therein have usually neutral hydrogen masses between $10^{9} M_{\odot}$ and few $10^{10} M_{\odot}$ (Fig. 2). The global properties of the main galaxies are listed in Table A.1.

\section{Results}

We ran our code on data-cubes smoothed to $30^{\prime \prime}$ and to $60^{\prime \prime}$. The results obtained with these two data sets are thoroughly comparable. We fixed a sigma-clipping threshold for the source finder equal to 4 (see Eq. (1)) and a secondary threshold for growing objects at the edges of 2.5. After extensive experiments, these values appeared the best compromise between reaching low sensitivities and avoiding spurious detections.

We found that, among 148 data-cubes, $101(\sim 68.2 \%)$ had no detectable companions, whereas $47(\sim 31.8 \%)$ contained multiple systems. Among these 47 data-cubes, $15(\sim 10.1 \%$ of the total, $\sim 31.9 \%$ of multiple systems) contained only galaxies with similar masses $\left(M_{\text {bar,sat }} / M_{\text {bar,main }}>0.20\right)$. Six data-cubes ( $\sim 4.1 \%$ of the total, $\sim 12.8 \%$ of multiple systems) show both major and minor companions, and 26 data-cubes $(\sim 17.6 \%$ of the total, $\sim 55.3 \%$ of multiple systems) show only dwarf companions. Overall, among 148 analysed data-cubes, $21(\sim 14.2 \%)$, show companions that might be possible candidates for a future major merging, while 32 data-cubes $(\sim 21.6 \%)$ show potential candidates for minor mergers. Some examples of spiral galaxies with minor satellites are shown in Fig. 3.

We focused on potential minor mergers, and all data-cubes with only major companions were excluded from the further analysis. For the six data-cubes with both types of companions, we assumed as the main galaxy the one with the largest 
E. M. Di Teodoro and F. Fraternali: Gas accretion from minor mergers in local spiral galaxies
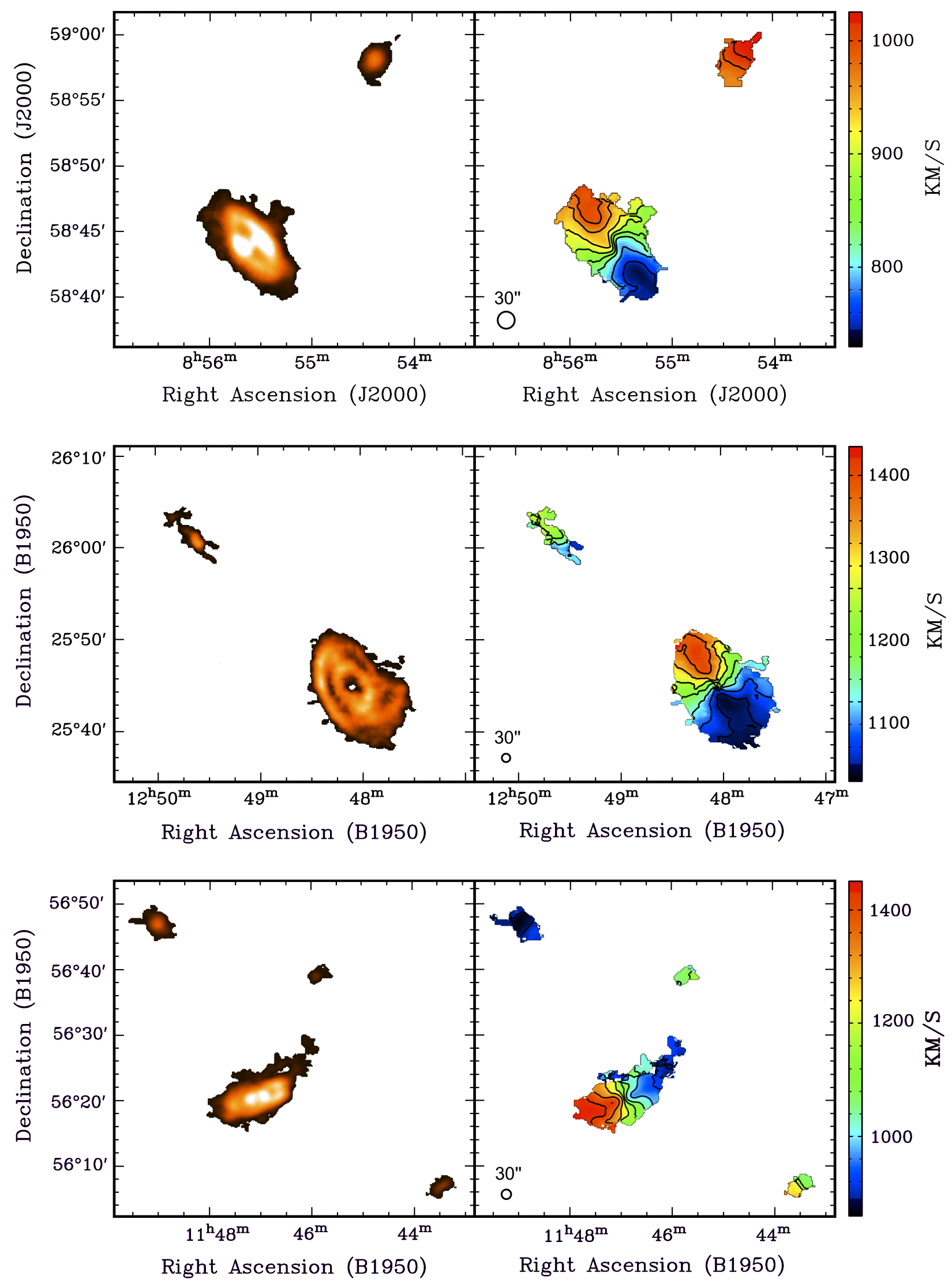

Fig. 3. Three examples of multiple systems in the WHISP sample. From top to bottom, UGC 4666, UGC 7989 , UGC 6787 and their dwarf companions. In the left panels, the HI column-density maps (0th moment), in the right panels, the velocity fields (first moment) obtained from $30^{\prime \prime}$ smoothed data-cubes. 
A\&A 567, A68 (2014)

Table 1. Detected companions of the WHISP spiral galaxies with $M_{\mathrm{bar}, \text { at }} / M_{\mathrm{bar}, \text { main }} \leq 0.20$.

\begin{tabular}{|c|c|c|c|c|c|c|c|c|c|}
\hline $\begin{array}{c}\text { Name } \\
\text { (1) }\end{array}$ & $\begin{array}{c}\text { Main galaxy } \\
\text { (2) }\end{array}$ & $\begin{array}{c}\text { Coord. (J2000) } \\
\text { RA-Dec } \\
\text { (3) }\end{array}$ & $\begin{array}{c}D \\
\mathrm{Mpc} \\
(4)\end{array}$ & $\begin{array}{c}v_{\text {sys }} \\
\mathrm{km} \mathrm{s}^{-1} \\
(5)\end{array}$ & $\begin{array}{c}w_{20} \\
\mathrm{~km} \mathrm{~s}^{-1} \\
(6)\end{array}$ & $\begin{array}{l}M_{\mathrm{HI}} \\
10^{8} M_{\odot} \\
\quad(7)\end{array}$ & $\begin{array}{c}d_{\text {proj }} \\
\text { kpc } \\
(8)\end{array}$ & $\begin{array}{c}t_{\text {coll }} \\
10^{8} \mathrm{yr} \\
(9)\end{array}$ & $\begin{array}{c}\dot{M}_{\mathrm{HI}} \\
M_{\odot} / \mathrm{yr} \\
(10)\end{array}$ \\
\hline AGC 102802 & UGC 485 & J004702.7+301243 & 58.9 & 5296 & 85 & 9.08 & 117 & 15.7 & 0.58 \\
\hline AGC 113996 & UGC 624 & $\mathrm{~J} 010107.2+304052$ & 78.3 & 4762 & 29 & 8.76 & 119 & 11.6 & 0.75 \\
\hline AGC 113884 & UGC 624 & J010000.3+302357 & 78.3 & 4717 & 96 & 5.04 & 391 & 17.8 & 0.28 \\
\hline [VH2008] J0101+4744 & UGC 625 & J010118.4+474432 & 28.3 & 2795 & 62 & 3.02 & 36 & 4.5 & 0.67 \\
\hline $\mathrm{DF} 1^{\dagger}$ & UGC 1437 & J015708.1+354825 & 54.5 & 4592 & 172 & 14.80 & 133 & 9.8 & 1.51 \\
\hline PGC 9994 & UGC 2141 & J030653.0+301542 & 24.7 & 812 & 43 & 7.37 & 75 & 5.6 & 1.32 \\
\hline PGC 2328690 & UGC 2459 & $\mathrm{~J} 030225.7+485452$ & 32.4 & 2449 & 134 & 16.29 & 131 & 14.3 & 1.14 \\
\hline [KLT2208] HI J0302+352* & UGC 2487 & J030210.5+351627 & 72.2 & 4967 & 55 & 15.43 & 351 & 18.1 & 0.85 \\
\hline [SOS2010] J0301491+3529012 & UGC 2487 & J030147.2+352839 & 72.2 & 4909 & 38 & 3.61 & 129 & 8.6 & 0.42 \\
\hline UGC 2813 & UGC 2800 & $\mathrm{~J} 034234.1+711828$ & $16.1^{1}$ & 1381 & 62 & 0.99 & 33 & 4.2 & 0.24 \\
\hline HFLLZOA G136.96+14.21 & UGC 2916 & J040403.5+713707 & 68.0 & 4450 & 158 & 14.36 & 114 & 10.8 & 1.33 \\
\hline 2MASX J04550438+3002212 & UGC 3205 & $\mathrm{~J} 045826.3+295653$ & 47.6 & 3239 & 173 & 10.26 & 350 & 17.6 & 0.58 \\
\hline $\mathrm{DF} 2^{\dagger}$ & UGC 3205 & $\mathrm{~J} 045504.2+300209$ & 47.6 & 3530 & 47 & 5.03 & 184 & 9.7 & 0.52 \\
\hline $\mathrm{DF}^{\dagger}$ & UGC 3205 & $\mathrm{~J} 045653.8+293602$ & 47.6 & 3229 & 110 & 19.34 & 385 & 18.3 & 1.06 \\
\hline $\mathrm{DF} 4^{\dagger}$ & UGC 3382 & $\mathrm{~J} 055903.3+621719$ & 67.2 & 4407 & 64 & 3.83 & 160 & 12.6 & 0.30 \\
\hline $\mathrm{DF}^{\dagger}$ & UGC 3407 & $\mathrm{~J} 060841.0+415647$ & 39.3 & 3683 & 66 & 3.50 & 96 & 10.1 & 0.35 \\
\hline $\mathrm{DF}^{\dagger}$ & UGC 3407 & $\mathrm{~J} 060913.3+420104$ & 39.3 & 3688 & 114 & 4.51 & 48 & 7.9 & 0.57 \\
\hline $\mathrm{DF}^{\dagger}$ & UGC 3407 & J060853.9+420338 & 39.3 & 3693 & 73 & 0.80 & 27 & 3.5 & 0.23 \\
\hline $\mathrm{DF}^{\dagger, *}$ & UGC 3422 & $\mathrm{~J} 061633.1+705743$ & 77.2 & 4009 & 24 & 3.22 & 238 & 9.8 & 0.33 \\
\hline GALEXASC J061256.68+710650.6 & UGC 3422 & J061254.8+710659 & 77.2 & 3998 & 104 & 5.52 & 83 & 7.5 & 0.74 \\
\hline NPM1G +60.0018 & UGC 3546 & $\mathrm{~J} 065150.2+604122$ & 17.9 & 1768 & 52 & 1.12 & 58 & 5.8 & 0.19 \\
\hline GALEXASC J070643.91+635521.0 & UGC 3642 & $\mathrm{~J} 070645.1+635515$ & 67.3 & 4714 & 106 & 3.56 & 169 & 11.1 & 0.32 \\
\hline UGC 3660 & UGC 3642 & $\mathrm{~J} 070634.1+635056$ & 67.3 & 4261 & 75 & 8.70 & 350 & 17.9 & 0.49 \\
\hline KUG 0829+227B & UGC 4458 & J083247.7+223443 & 68.6 & 4621 & 231 & 12.80 & 105 & 12.4 & 1.03 \\
\hline MCG +10-13-030 & UGC 4666 & J085422.1+585908 & 16.0 & 1016 & 90 & 1.26 & 69 & 5.7 & 0.22 \\
\hline SDSS J091001.72+325659.8 & UGC 4806 & J091005.0+325607 & 25.5 & 2021 & 125 & 4.23 & 89 & 9.5 & 0.45 \\
\hline KUG $0906+333 \mathrm{~A}$ & UGC 4806 & J090919.5+330734 & 25.5 & 1856 & 60 & 1.88 & 18 & 5.7 & 0.33 \\
\hline SDSS J093137.13+292533.3 & UGC 5060 & J093138.0+292534 & 24.0 & 1608 & 77 & 0.53 & 117 & 9.9 & 0.05 \\
\hline KDG 059 & UGC 5253 & J095156.6+720439 & 16.6 & 1121 & 46 & 2.03 & 57 & 6.2 & 0.33 \\
\hline UGC 6797 & UGC 6778 & $\mathrm{~J} 114940.5+482533$ & 17.1 & 962 & 81 & 7.28 & 87 & 8.5 & 0.86 \\
\hline SDSS J115027.42+490105.9 & UGC 6778 & $\mathrm{~J} 115027.4+490106$ & 17.1 & 1120 & 31 & 1.67 & 138 & 11.2 & 0.12 \\
\hline UGC 6791 & UGC 6786 & $\mathrm{~J} 114923.6+264428$ & $22.5^{1}$ & 1866 & 274 & 5.38 & 111 & 10.0 & 0.54 \\
\hline SDSS J114820.16+562045.7 & UGC 6787 & $\mathrm{~J} 114820.6+562049$ & 22.1 & 1080 & 28 & 0.62 & 105 & 7.6 & 0.08 \\
\hline UGC 6733 & UGC 6787 & $\mathrm{~J} 114535.7+555313$ & $19.1^{2}$ & 1158 & 187 & 5.26 & 130 & 10.3 & 0.51 \\
\hline UGC 6816 & UGC 6787 & $\mathrm{~J} 115047.5+562719$ & $17.1^{1}$ & 887 & 115 & 5.78 & 146 & 11.0 & 0.52 \\
\hline SDSS J122442.59+544441.3 & UGC 7506 & $\mathrm{~J} 122440.2+544448$ & 36.0 & 2495 & 109 & 2.18 & 154 & 11.6 & 0.19 \\
\hline UGC 8005 & UGC 7989 & $\mathrm{~J} 125149.1+254644$ & $14.3^{1}$ & 1196 & 198 & 8.84 & 101 & 8.6 & 1.02 \\
\hline UGC 8254 & UGC 8307 & $\mathrm{~J} 131038.2+363807$ & 19.1 & 1088 & 105 & 3.71 & 149 & 16.1 & 0.23 \\
\hline $\mathrm{DF}^{\dagger, *}$ & UGC 8307 & $\mathrm{~J} 131153.6+362758$ & $19.1^{1}$ & 954 & 75 & 1.92 & 100 & 14.2 & 0.14 \\
\hline UGC 8271 & UGC 8307 & $\mathrm{~J} 131131.3+361655$ & $18.5^{1}$ & 1145 & 150 & 6.99 & 156 & 22.1 & 0.32 \\
\hline $\mathrm{DF} 10^{\dagger, *}$ & UGC 8307 & $\mathrm{~J} 131134.3+362942$ & 19.1 & 1191 & 32 & 0.58 & 109 & 15.5 & 0.04 \\
\hline KUG $1309+362$ & UGC 8307 & $\mathrm{~J} 131146.7+355731$ & 19.1 & 1123 & 26 & 0.30 & 245 & 24.9 & 0.01 \\
\hline UGC 8303 & UGC 8307 & $\mathrm{~J} 131317.6+361303$ & $18.5^{1}$ & 948 & 92 & 9.77 & 139 & 20.3 & 0.48 \\
\hline UGC 8314 & UGC 8307 & $\mathrm{~J} 131401.0+361908$ & 19.1 & 938 & 71 & 1.06 & 113 & 21.2 & 0.05 \\
\hline MCG +08-27-001 & UGC 9366 & $\mathrm{~J} 143359.2+492647$ & 38.9 & 2122 & 127 & 6.18 & 88 & 6.2 & 1.00 \\
\hline KUG $1512+557$ & UGC 9797 & $\mathrm{~J} 151400.2+553222$ & 46.6 & 3550 & 154 & 9.66 & 94 & 9.4 & 1.03 \\
\hline SDSS J152617.51+404004.0 & UGC 9858 & $\mathrm{~J} 152617.9+404008$ & 32.2 & 2687 & 51 & 11.80 & 66 & 7.7 & 1.53 \\
\hline MCG +08-34-005 & UGC 11283 & $\mathrm{~J} 183400.4+492233$ & 30.0 & 2076 & 63 & 3.75 & 51 & 7.5 & 0.50 \\
\hline GALEXASC J215645.61+275419.5 & UGC 11852 & $\mathrm{~J} 215645.7+275418$ & 82.4 & 5710 & 46 & 5.30 & 221 & 15.2 & 0.35 \\
\hline ZOAG G095.92-08.72 & UGC 11951 & $\mathrm{~J} 221145.4+453649$ & 14.2 & 1145 & 78 & 7.52 & 74 & 8.3 & 0.91 \\
\hline
\end{tabular}

Notes. (1) First name in NED archive or DF if not classified; (2) UGC name of the main galaxy; (3) celestial coordinates; (4) adopted distance (same as the main galaxy or taken from EDD catalogue, when specified); (5) systemic velocity; (6) line width of the global profile at the $20 \%$ level; (7) total HI mass; (8) projected distance from the main galaxy; (9) time of collision with the main galaxy in a parabolic orbit; and (10) gas accretion rate onto the main galaxy. ${ }^{(\dagger)}$ Not catalogued in the NED, HyperLEDA, or SIMBAD archives. ${ }^{(*)}$ Without a clear optical/UV counterpart in DSS, SDSS or GALEX images. DF8 is not covered by the SDSS survey. ${ }^{(1)}$ Distance from EDD catalogue.

HI mass and ignored the other spiral galaxies. Fifty dwarf gasrich satellites are detected in total (Table 1). Forty-six dwarf galaxies have a clear optical counterpart in the Sloan Digital Sky Survey (SDSS) or in the Digitized Sky Survey (DSS) images.
Four detections, marked with an asterisk in Table 1, are not unambiguously identifiable and might be either very faint dwarf galaxies or HI clouds. Most satellites are already catalogued in galaxy archives. Ten galaxies, marked with a dagger in Table 1, 

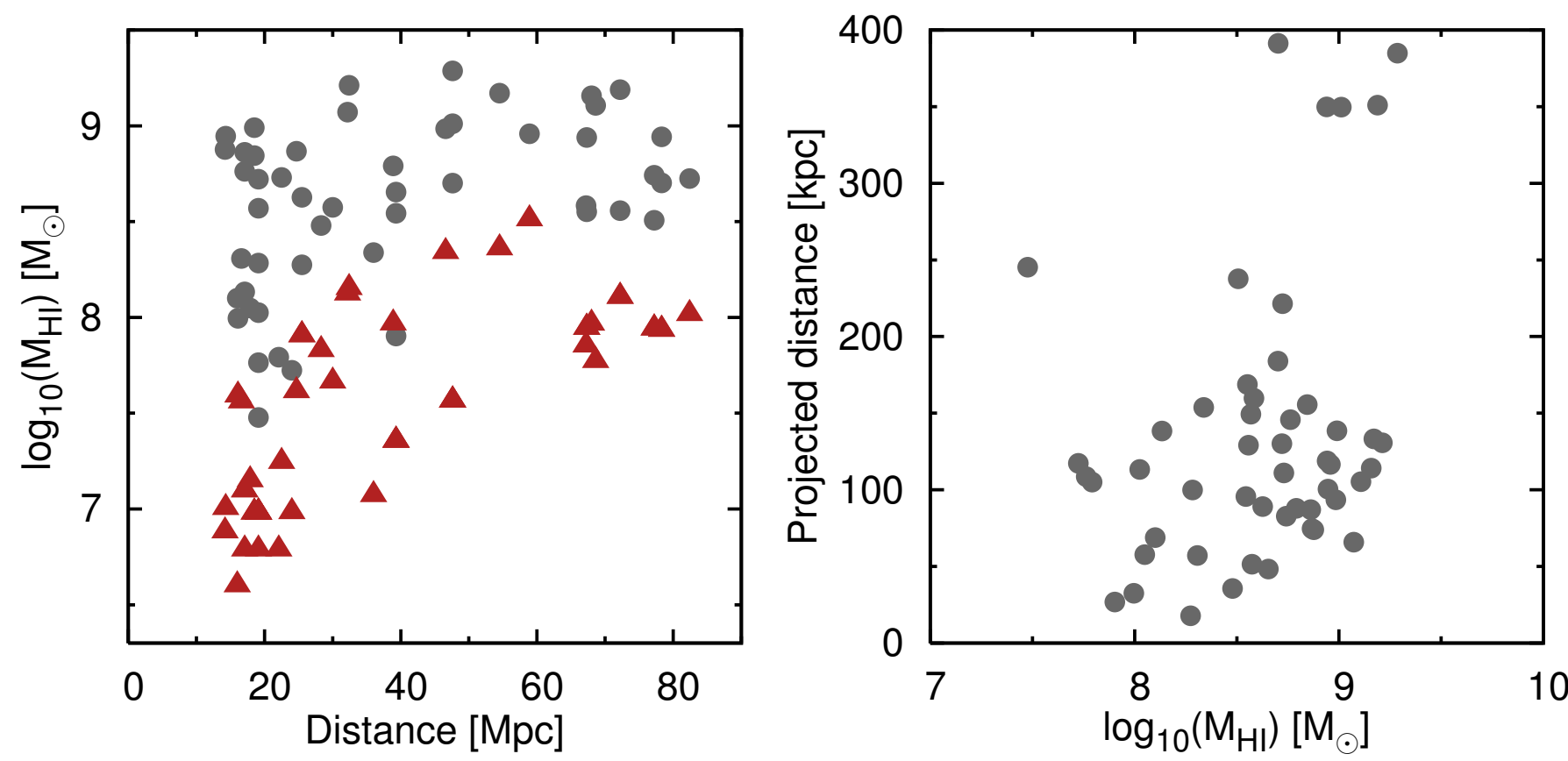

Fig. 4. Left panel, grey dots: HI masses of detected companions as a function of distance; red triangles: lowest detectable mass in the corresponding data-cubes. Right panel: distance from the main galaxies projected onto the plane of the sky of the detected satellites as a function of their HI mass.

do not seem to be catalogued. The Hi masses of the detected dwarf galaxies vary between about $10^{7} M_{\odot}$ and few $10^{9} M_{\odot}$. The HI mass distribution of the main galaxies and their minor satellites is shown in Fig. 2. The mass function for spiral galaxies is peaked at $\log M_{\mathrm{HI}\left[M_{\odot}\right]} \sim 9.5$, consistently with studies on wider HI samples (e.g., Zwaan et al. 2005). Most dwarf companions have masses of a few $10^{8} M_{\odot}$ and their mass distribution has a cut-off above $5 \times 10^{9} M_{\odot}$. This is partially due to our selection criteria. However, it is interesting to note that this distribution is fairly similar to that of HI-rich dwarf galaxies in the Local Group and in Local Group analogues (e.g., Grcevich \& Putman 2009; Pisano et al. 2011). In Fig. 4 we show the HI masses of the detected dwarf galaxies as a function of the distance from the Milky Way. The red triangles represent the minimum detectable mass for each data-cube, calculated using Eq. (5) on a 3D region with the size of a spatial beam times the velocity resolution (two channels) and a flux of $4 \times$ rms noise of the cube. This is the minimum mass that an object must have to be accepted by the source-finding algorithm. Note the bias effect on the detectable mass due to the distance (see discussion in Sect. 4.1).

The projected distances of the dwarf satellites from the main galaxies typically range from some dozens to a few hundred kiloparsecs, and typical time-scales for collisions, estimated through the parabolic orbit approximation, are between $\sim 100 \mathrm{Myr}$ and $2 \mathrm{Gyr}$. The number of dwarfs within $100 \mathrm{kpc}$ from the main galaxies and between 100 and $200 \mathrm{kpc}$ is almost the same. In the right panel of Fig. 4 we show the projected distance as a function of the dwarf HI masses. Within $200 \mathrm{kpc}$, dwarf galaxies are quite uniformly distributed over the HI masses. There is a weak tendency for companions to be more massive at larger distances, as we may expect. However, there is an observational bias that can affect this plot. It is a combination of two effects: the linear field-of-view of the observations increases with distance, while the minimum detectable mass (Fig. 4, left panel) and the linear resolution decrease. Thus we may detect preferentially companions with lower masses closer to the main galaxies, and vice versa. Moreover, there is also a selection effect due to the primary beam attenuation, that is, at large angular distances, only massive systems are detected because of the lower sensitivity of the instrument. These effects make it difficult to compare our findings with studies of dwarfs galaxies in the Local Group.

The systemic velocity of dwarf galaxies is calculated as the average midpoint between the velocities at the $20 \%$ and $50 \%$ of the peak flux of their global Hi profiles. The $\Delta v_{\text {sys }}=$ $\left\|v_{\text {sys,main }}-v_{\text {sys,sat }}\right\|$ ranges between a few tens to a few hundred $\mathrm{km} \mathrm{s}^{-1}$. Satellites do not have systemic velocities that differ by more than $300 \mathrm{~km} \mathrm{~s}^{-1}$ from those of the main galaxies. The velocity widths $w_{20}$ of dwarf galaxies, taken at the $20 \%$ of the peak flux, are usually lower than $200 \mathrm{~km} \mathrm{~s}^{-1}$, even if corrected for a mean inclination of 60 degrees, except for three galaxies. Overall, most of the satellites have $w_{20}<100 \mathrm{~km} \mathrm{~s}^{-1}$.

For each data-cube with identified dwarf companions, we calculated the maximum possible accretion rate of cold hydrogen gas $\dot{M}_{\mathrm{HI}}$ onto the main galaxy, the star formation rate $\dot{M}_{\mathrm{SF}}$ of the main galaxy, and the ratio $\dot{M}_{\mathrm{HI}} / \dot{M}_{\mathrm{SF}}$. For all galaxies, with or without identified companions, a potentially hidden accretion from dwarfs below the detectability limit was estimated. The hidden accretion rate was calculated by dividing the above-mentioned minimum detectable mass by the average collision time over the sample, that is, $1.1 \mathrm{Gyr}$. Integrating the HI mass function $\left(\phi_{*}\left[\mathrm{Mpc}^{-3} \mathrm{dex}^{-1} h_{70}^{3}\right]=4.8 \pm 0.3 \times 10^{3}\right.$, $\log \left(M_{*} / M_{\odot}\right)+2 \log h_{70}=9.96 \pm 0.02$ and $\alpha=-1.33 \pm 0.02$, Martin et al. 2010) below the detection limit and within the volume of each data-cube always gives HI masses lower than minimum detectable mass. Thus, with our choice we maximize the mass of the undetected galaxies.

We found a mean upper limit for the accretion in galaxies with identified minor companions of $0.86 M_{\odot} \mathrm{yr}^{-1}$, with a mean ratio $\left\langle\dot{M}_{\mathrm{HI}} / \dot{M}_{\mathrm{SF}}\right\rangle \sim 0.67$. A more meaningful estimate is the mean upper limit to the accretion over the whole sample, however, which is $\dot{M}_{\mathrm{HI}}=0.28 M_{\odot} \mathrm{yr}^{-1}$ compared with the average star formation rate of $1.29 M_{\odot} \mathrm{yr}^{-1}$, with a mean ratio $\left\langle\dot{M}_{\mathrm{HI}} / \dot{M}_{\mathrm{SF}}\right\rangle \sim 0.22$. The median of $\dot{M}_{\mathrm{HI}} / \dot{M}_{\mathrm{SF}}$ is 0.07 . Thus, 


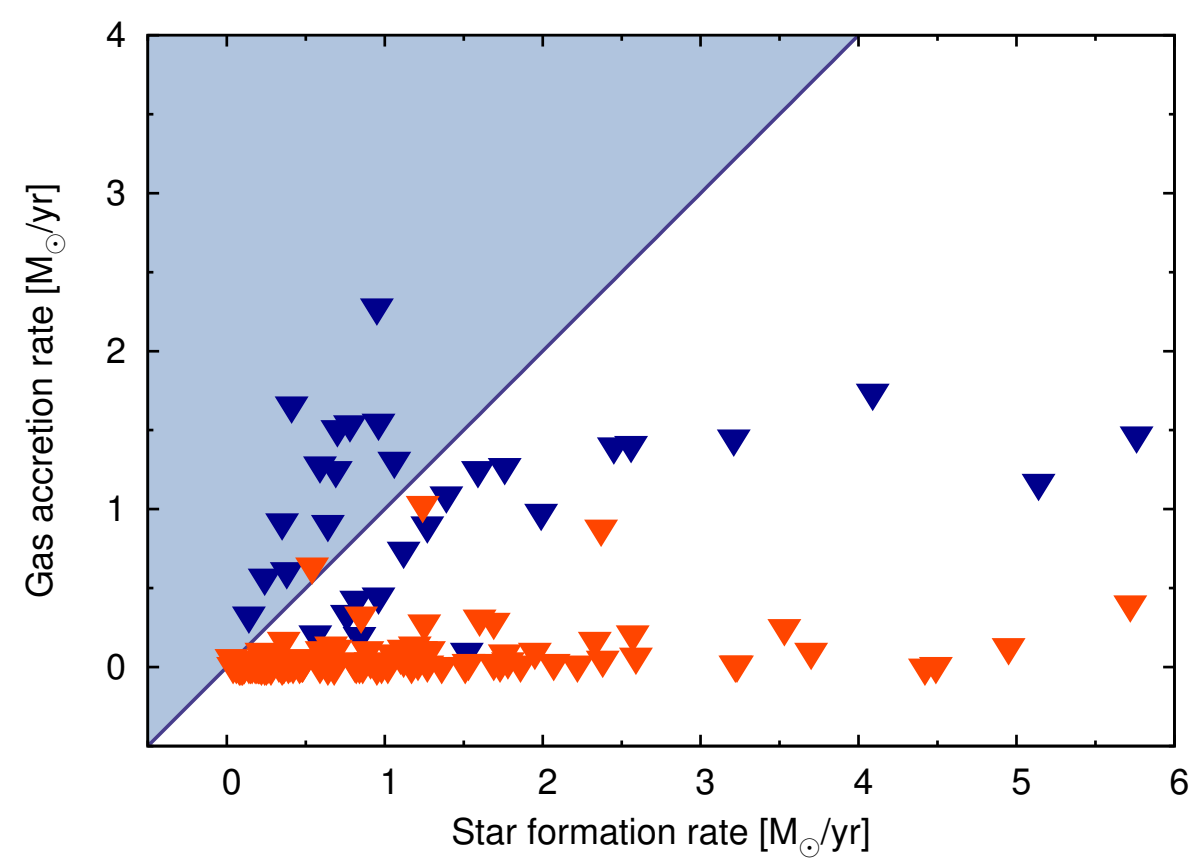

Fig. 5. Upper limits to the cold gas accretion rates from satellites vs star formation rates in spiral galaxies in the WHISP sample. The inverted dark blue triangles are upper limits to the gas accretion rate for galaxies with detected satellites (including both visible and hidden accretion), the inverted orange triangles are the hidden accretion upper limits for galaxies without companions, estimated as discussed in the text. The star formation rates are lower limits calculated from the far-infrared fluxes. The blue-shadowed region represents a complete feeding of SF through minor mergers. the ratio of the gas needed for star formation to the maximum gas accretion provided by minor mergers is between 5 and 14 . Considering a fraction of gas recycled from stellar feedback of $30 \%$ (e.g. Naab \& Ostriker 2006) leads to a ratio between 3 and 10 .

These results show that the number of dwarf galaxies in the local Universe is on average too low to guarantee the continuous gas replenishment needed by star formation. In Fig. 5 we show a plot of $\dot{M}_{\mathrm{HI}}$ versus $\dot{M}_{\mathrm{SF}}$ for each galaxy individually. If the gas accretion were high enough to sustain the star formation of the main galaxies, the data points would have fully populated the blue-shadowed region in the upper-left corner, whereas the vast majority lie well below the blue straight-line, indicating a ratio $\dot{M}_{\mathrm{HI}} / \dot{M}_{\mathrm{SF}}=1$. We conclude that minor mergers cannot add enough gas to the discs and sustain star formation. Once again, our values of gas accretion rates are very strong upper limits because of our very stringent assumptions, and the real accretion rates might reasonably be one order of magnitude lower than our estimate. Incidentally, we note that our assumptions would imply that all dwarf galaxies disappear in the next 2 Gyr. We stress that our SFRs are very likely lower limits because they were calculated using only FIR fluxes. This bias goes in the direction of strengthening our findings.

We repeated the analysis of the WHISP data-cubes using a sigma-clipping threshold for the sources of $3 \sigma$ and $5 \sigma$. Reducing the detection threshold leads the program to identify many more dwarf companions: more than 100 minor satellites are detected at the lower level, but most of these sources are clearly false detections and the results obtained are very likely unreliable. Such a large fraction of wrong detections is probably due to the low signal-to-noise of the WHISP data-cubes. Instead, increasing the detection threshold to $5 \sigma$ leads to results very similar to those described above as just two of the dwarf companions found at $4 \sigma$ are missed by the rejection criteria, namely the satellites of UGC 7506 and UGC 9858. These companions are actually good detections, as quoted in the literature (Noordermeer et al. 2005), but at a $5 \sigma$ level they are discarded by the one-beam covering requirement. The mean values of the accretion rate at $5 \sigma$ also agree with those found at $4 \sigma$.

\section{Discussion}

The application of our code to the WHISP catalogue led to a firm upper limit for the accretion of cold gas from minor mergers in the local Universe of $0.28 M_{\odot} \mathrm{yr}^{-1}$. The total multiple system fraction for the WHISP sample is $\sim 32 \%$, in particular, $\sim 22 \%$ of galaxies are accompanied by minor companions and $\sim 14 \%$ are major systems. Here we discuss the main uncertainties of our results and their relevance.

\subsection{Uncertainties}

Our estimate does not take into account the molecular fraction. The amount of molecular gas in dwarf galaxies is highly unconstrained as they are often undetected in $\mathrm{CO}$ emission lines (e.g., Taylor et al. 1998). They also usually have low metallicities, making the conversion between $\mathrm{CO}$ and $\mathrm{H}_{2}$ even more uncertain (e.g., Boselli et al. 2002). However, any realistic correction for molecular gas is not expected to increase our accretion rate by more than a factor two.

WHISP is a source-targeted survey, and it can obviously not be considered as a complete sample. The selection criterion, based on the apparent size of the observed galaxies, produces a catalogue that favours progressively larger and more massive galaxies moving to greater distances from the Milky Way. This effect can be appreciated in Fig. 4 (left panel), although it appears to be not too severe. The growth with the distance of the minimum detectable mass furthermore makes it impossible to detect low-mass satellites at large distances. To test the importance of these biases, we considered only datacubes with a minimum detectable mass $M_{\text {det }} \leq 10^{8} M_{\odot}$. In this way, we obtained a sub-sample of galaxies where satellites are quite uniformly distributed over the mass and the distance ranges (left panel of Fig. 4). The highest accretion rate obtained in this case is $0.21 M_{\odot} \mathrm{yr}^{-1}$. Reducing the threshold to data-cubes with $\log M_{\text {det }} \leq 5 \times 10^{7} M_{\odot}$ leads to a highest accretion rate of $0.18 M_{\odot} \mathrm{yr}^{-1}$. These values indicate that our accretion rate estimates are not strongly affected by the incompleteness of the dwarf galaxy sample. 
Another bias effect is related to the linear field of view, which is greater at larger distances. In the farthest systems, the field of view allows us to observe satellites with projected distances of some hundred kpc from the main galaxies, whereas we cannot go beyond one hundred $\mathrm{kpc}$ in the closest systems. The primary beam attenuation of the WSRT is significantly large ( $~ 80 \%$ of the flux is missed) beyond $25^{\prime}$ from the pointing centre, corresponding to $\sim 70 \mathrm{kpc}$ at about $10 \mathrm{Mpc}$. This indicates that we should be able to also detect fairly well-separated satellites in the nearest systems. The most distant satellites have longer collision time-scales, and their contribution to the global accretion is expected to be smaller. In our sample, considering only satellites within $100 \mathrm{kpc}$ from the main galaxies gives an accretion rate of $0.38 M_{\odot} \mathrm{yr}^{-1}, 0.27 M_{\odot} \mathrm{yr}^{-1}$ between 100 and $200 \mathrm{kpc}$ and $0.21 M_{\odot} \mathrm{yr}^{-1}$ beyond $200 \mathrm{kpc}$ (the global value being $0.86 M_{\odot} \mathrm{yr}^{-1}$ ). These results show that the contribution of very distant satellites is progressively less important, thus the limited field of view of the closest systems probably does not significantly affect our accretion rate estimate.

In the literature, mergers are usually classified on the basis of their dynamical mass ratio: pairs of galaxies with $M_{\text {sat }} / M_{\text {main }} \leq$ $0.1-0.2$ are considered minor mergers, otherwise they are major mergers. Unfortunately, we cannot easily estimate the dynamical masses of satellite galaxies from the HI data. Thus, in this work, we divided satellites according to the ratio of their baryonic mass to the main galaxy baryonic mass. Satellites with baryonic content lower than $20 \%$ of the main galaxies $\left(M_{\text {bar,sat }} / M_{\text {bar,main }} \leq\right.$ $0.20)$ are classified as minor companions. This is an arbitrary but conservative choice, since most detected satellites have a mass ratio $\ll 0.05$. It is interesting to quantify the accretion rate using different baryonic mass ratios, however. In our sample, the highest accretion rates range between $0.20 M_{\odot} \mathrm{yr}^{-1}$ for $M_{\text {bar,s }} / M_{\text {bar, },} \leq 0.1$ and $0.56 M_{\odot} \mathrm{yr}^{-1}$ for $M_{\text {bar,sat }} / M_{\text {bar,main }} \leq 0.5$. If we consider all galaxy pairs as potential mergers and calculate the accretion rate by accreting the less massive ones onto the most massive ones, we obtain a value of $1.22 M_{\odot} \mathrm{yr}^{-1}$. Even this excessive overestimate is of the same order as the mean SFR. We conclude that mergers in the local Universe cannot sustain the star formation in spiral galaxies.

\subsection{Comparison with other estimates}

The accretion of cold gas from minor mergers in the local Universe has been estimated by Sancisi et al. (2008), who visually inspected and compared total maps, velocity fields, and position-velocity diagrams for the WHISP galaxies. They found a minor-merger fraction of about $25 \%$. Unlike our approach, they considered only systems that showed clear signs of tidal interactions, such as tails, bridges, disturbed HI morphologies, and/or kinematics. By assuming typical HI masses of the dwarfs of the order $10^{8-9} M_{\odot}$ and a lifetime for observed features of about 1 Gyr, Sancisi et al. inferred a mean accretion rate of HI gas around $0.1-0.2 M_{\odot} \mathrm{yr}^{-1}$ and stressed that this value is most likely a lower limit. It is worth noting that most systems we considered as potential minor mergers were not recognized by Sancisi et al. and, conversely, many interactions they identified were not found by our code. The reason is simple: our code searches for "separated" objects and treats all dwarf companions as candidates for minor mergers, including those that show no signs of ongoing interaction. In other words, we consider the population of dwarfs in the environment of a spiral galaxy that could become a minor merger in the near future. Our code identifies companions until the two galaxies start "touching" each other, and we estimate the accretion rate using the time-scale for collision as accretion time. Instead, Sancisi et al. (2008) found a later stage of merging, that is, when galaxies are strongly interacting and the gas is visibly disturbed in the morphology and/or in the kinematics. Consequently, they calculated the accretion rate using as time-scale the dynamical time that it should take for these features to disappear as the gas redistributes uniformly in the disc. In our work the accretion process ends when galaxies touch each other, whereas for Sancisi et al. (2008) that was the starting point. However, since the population of dwarf galaxies has most likely remained similar in the past Gyr or so, the two accretion rates should be similar. Interestingly, our upper limit of $\dot{M}_{\mathrm{HI}}<0.28 M_{\odot} \mathrm{yr}^{-1}$ does not contradict the average accretion rate estimated by Sancisi et al. (2008).

\subsection{Merger fraction}

Most studies recently published on the local merging systems have been made using images from optical-UV galaxy surveys (e.g., Patton et al. 2000; Lambas et al. 2012; Robotham et al. 2012) such as the Second Redshift Survey of Southern Sky (SRSS2), the Sloan Digital Sky Survey (SDSS) and the recent Galaxy And Mass Assembly (GAMA) survey, whereas just a few studies have been carried out using HI data (Sancisi et al. 2008; Holwerda et al. 2011). These studies have mainly investigated the fraction and the rate (fraction of mergers per comoving volume and time units) of galaxies showing signs of interactions and their evolution with time.

To date, two main approaches have been used to estimate the galaxy merger fraction, and both made use of high-resolution imaging. The pair method consists of counting the galaxies that are spatially separated from each other by less than a few tens of kpc and with spectroscopic radial velocities that do not differ by more than a few hundreds of $\mathrm{km} \mathrm{s}^{-1}$ (e.g., Le Fèvre et al. 2000; Lin et al. 2008). Using this type of approach, it is possible to estimate a "progenitor galaxy" merger fraction. The second approach identifies mergers by quantifying morphological signatures that can be related to past or ongoing interactions, such as asymmetries and/or tails. This method makes use of several parameters to describe peculiar light distributions, such as the concentration-asymmetry-smoothness parameters (CAS, Conselice 2003) or the Gini-M 20 parameters (Lotz et al. 2004). This technique can identify mergers in a relatively late stage, but not all asymmetric galaxies are necessarily merger features. The asymmetry method is similar to the technique used by Sancisi et al. (2008), whereas our approach on HI data-cubes is conceptually similar to the close-pairs method. The main difference is that we do not impose any limit for the projected distance between galaxies, whereas the velocity criterion is implicit in the data-cubes. Moreover, we select objects in 3D space (so potentially also overlapping in the sky) and we independently estimate the shortest collision time for each galaxy.

The asymmetry and close-pairs methods have been widely used with optical galaxy surveys, but, despite the large number of studies, there is little consensus on the galaxy merger rate and its evolution with redshift. Current observations of the fraction of galaxy undergoing a merger differ by an order of magnitude, from 2\% (e.g., Patton et al. 2000; De Propris et al. 2007, $2.3 \%$ and $1.9 \%$, respectively) to $15 \%$ (e.g., de Ravel et al. 2009), and its trend with redshift varies from no evolution (e.g., Jogee et al. 2009) to strong evolution (e.g., López-Sanjuan et al. 2009). These discrepancies mainly arise from the different criteria for galaxy counting, merger selection, and bias in the galaxy samples. The value we found $(\sim 32 \%)$ is a companion fraction rather than a merger fraction as some companions that we considered 
are fairly far away from the main galaxies (Fig. 4, right panel). It is therefore difficult to compare our fraction with the abovementioned values. Broadly speaking, our estimate, which is indeed an upper limit, is higher at least by a factor 2-3 because our program treats all multiple systems as mergers and, working with HI data, more easily identifies dwarf gas-rich companions than is possible from optical observations. However, if we exclude the very far away companions, namely those beyond $100 \mathrm{kpc}$ of projected distance, we obtain a companion fraction of $\sim 14 \%$, not too different from the values found from optical studies. Finally, we stress that the WHISP sample is insignificant compared with other local references based on large catalogues, such as the SDSS or the Millennium Galaxy Catalogue (MGC), so that our values are less reliable from a statistical point of view.

A recent study carried out by Holwerda et al. (2011) estimated the merger fraction and rate for the whole WHISP sample using both the close-pairs and asymmetry methods on HI total maps. Holwerda et al. found a merger fraction of $7 \%$ based on pairs, and $13 \%$ based on disturbed morphology. We cannot compare our merger fraction with the latter value, because our program ignores the galaxy morphology, but the former value is fully comparable and our estimate is significantly higher by about a factor 4 . A possible reason for this discrepancy is that Holwerda et al. based their pair fraction on 24 multiple systems previously identified and classified as interacting by Noordermeer et al. (2005b) and Swaters et al. (2002b), whereas our code detected many more satellites (see Table 1). If we use this sub-sample, the merger fractions become very similar.

\subsection{Other channels for gas accretion}

How star-forming galaxies can sustain their star formation is still an open question. In this study, we demonstrated that gas-rich minor mergers do not play a primary role and other dominant accretion channels must be considered. A way to fill the discrepancy between the estimated accretion rates and the SFRs could be to assume that the HI mass function were much steeper in the recent past than now, so that the number of dwarf satellites to be accreted were much higher. However, to date, no observational evidence in that direction can be achieved with the present generation of radio-telescopes, and the studies of the damped Lyman- $\alpha$ systems show a remarkable constancy of the HI mass throughout the Hubble time (e.g., Prochaska \& Wolfe 2009). Another possibility is that most accretion is supported by infalling of gas clouds with HI masses of $10^{7}-10^{6} M_{\odot}$, but recent deep observations of nearby groups of galaxies (e.g., Pisano et al. 2007; Chynoweth et al. 2009) as well as large blind surveys such as ALFALFA (Giovanelli et al. 2007) showed no evidence for a significant population of these small HI clouds. Moreover, studies of the Milky Way high-velocity clouds (HVCs) estimated a contribution to the total gas accretion of $0.1-0.2 M_{\odot} \mathrm{yr}^{-1}$ (e.g., Wakker et al. 2007; Putman et al. 2012), a value much lower than the SFR. In addition, the gas in the ionized phase could produce an additional accretion rate of $\sim 1 M_{\odot} \mathrm{yr}^{-1}$ (e.g., Shull et al. 2009), but it is not understood whether this gas can feed the star formation process in the disc. Numerical simulations (e.g., Fernàndez et al. 2012) support the idea that most of the gas infall in Milky Way-like galaxies is continuously provided by a drizzle and filamentary cosmological accretion, which would be almost undetectable or very difficult to identify (e.g., Lehner et al. 2013; Tumlinson et al. 2013). Finally, large amounts of matter could be supplied by the coronal gas cooling potentially triggered by supernova feedback (Marinacci et al. 2010).

\section{Conclusions and future prospects}

We estimated the maximum accretion of cold gas from minor mergers in a sample of large spiral galaxies from the WHISP catalogue. We used a source-finding algorithm to detect dwarf HI-rich satellites around these spiral galaxies and assumed that they will disappear and merge with the main galaxies in the shortest possible time. We found that $\sim 22 \%$ of galaxies have detected dwarf companions $\left(M_{\text {bar,sat }} / M_{\text {bar,main }} \leq 0.20\right)$ and estimated a maximum gas accretion rate onto the main galaxies over the whole sample of $0.28 M_{\odot} \mathrm{yr}^{-1}$. Given these assumptions, this value is a strong overestimate and the actual value can easily be an order of magnitude or more lower. From farinfrared luminosities, we calculated a mean star formation rate of $1.29 M_{\odot} \mathrm{yr}^{-1}$, which is nearly five times higher than the maximum gas accretion rate. These results strongly suggest that minor mergers cannot bring enough gas to guarantee a long-lasting star formation process in the discs of the spiral galaxies. We note that our method can also detect, if present, large floating HI clouds and include them in the accretion budget. We did not find any significant population of these clouds. Thus, most of the gas accretion seems to be hidden from the current investigations in HI emission.

WHISP is a fairly large sample of nearby galaxies, but it is very small compared with surveys carried out at other wavelengths. The new generation of radio telescopes, such as the SKA (Carilli \& Rawlings 2004) and its pathfinders, ASKAP (Johnston et al. 2008) and MeerKAT (Booth et al. 2009) and the restyling of existing interferometers, such as the WSRT with the APERTIF system (Verheijen et al. 2008) and the Karl G. Jansky VLA, will much increase the number of available data samples. In the next future, already scheduled HI surveys, such as WALLABY and DINGO with ASKAP, LADUMA with MeerKAT and WNSHS with WSRT/APERTIF, will increase the number of galaxies observed with radio interferometers by three orders of magnitude, from a few hundreds to about $10^{5}$. It will be very interesting to apply the type of analysis performed in this paper to these large galaxy samples.

Acknowledgements. We thank Renzo Sancisi, Tom Oosterloo, Thijs van der Hulst and Micol Bolzanella for helpful suggestions and fruitful discussions. E.d.T. personally thanks Gabriele Pezzulli for his help. We used the WHISP data sample and the EDD, NED, HyperLEDA and Simbad catalogues. This research made use of some parts of the Duchamp code, produced at the Australia Telescope National Facility, CSIRO, by Matthew Whiting. We acknowledge financial support from PRIN MIUR 2010-2011, project "The Chemical and Dynamical Evolution of the Milky Way and Local Group Galaxies", prot. 2010LY5N2T.

\section{References}

Aumer, M., \& Binney, J. J. 2009, MNRAS, 397, 1286

Bell, E. F., McIntosh, D. H., Katz, N., \& Weinberg, M. D. 2003, ApJS, 149, 289 Bertone, S., \& Conselice, C. J. 2009, MNRAS, 396, 2345

Bigiel, F., Leroy, A., Walter, F., et al. 2011, ApJ, 730, L13

Binney, J., \& Merrifield, M. 1998, Galactic Astronomy (Princeton NJ: Princeton University Press)

Bond, J. R., Cole, S., Efstathiou, G., \& Kaiser N. 1991, ApJ, 379, 440

Boselli, A., Lequeux, J., \& Gavazzi, G. 2002, A\&A, 384, 33

Booth, R. S., de Blok, W. J. G., Jonas, J. L., \& Fanaroff, B. 2009 [arXiv: 0910.2935]

Bregman, J. N. 2007, ARA\&A, 45, 221

Naab, T., \& Ostriker, J. P. 2006, MNRAS, 366, 899

Carilli, C. L., \& Rawlings, S. 2004, New Astron. Rev., 48, 979

Chiappini, C., Matteucci, F., \& Gratton, R. 1997, ApJ, 477, 765

Chomiuk, L., \& Povich, M. S. 2011, AJ, 142, 197

Chynoweth, K. M., Langston, G. I., Holley-Bockelmann, K., \& Lockman, F. J. 2009, AJ, 138, 287

Conselice, C. J. 2003, ApJS, 147, 1 
E. M. Di Teodoro and F. Fraternali: Gas accretion from minor mergers in local spiral galaxies

Dekel, A. \& Birnboim, Y. 2006, MNRAS, 368, 2

De Propris, R., Conselice, C. J., Liske, J., et al. 2007, ApJ, 666, 212 de Ravel, L., Le Fèvre, O., Tresse, L., et al. 2009, A\&A, 498, 379

Fernàndez, X., Joung, M. R., \& Putman, M. E. 2012, ApJ, 749, 181

Fraternali, F. 2010, AIPC, 1240, 135

Fraternali, F., \& Binney, J. J. 2008, MNRAS, 386, 935

Fraternali, F., \& Tomassetti, M. 2012, MNRAS, 426, 2166

Fraternali, F., van Moorsel, G., Sancisi, R., \& Oosterloo, T. 2002, AJ, 123, 3124

Grcevich, J., \& Putman, M. E. 2009, ApJ, 696, 385

Jogee, S., Miller, S. H., Penner, K., et al. 2009, ApJ, 697, 197

Johnston, S., Taylor, R., Bailes, M., et al. 2008, Exp. Astron., 22, 151

Kazantzidis, S., Zentner, A. R., Kravtsov, A. V., Bullock, J. S., \& Debattista, V. P. 2009, ApJ, 700, 1896

Kennicutt, R. C. 1998, ApJ, 498, 541

Kereš, D., Katz, N., Fardal, M., Davé, R., \& Weinberg, D. H. 2009, MNRAS, 395,160

Giovanelli, R., Haynes, M. P., Kent, B. R., et al. 2007, AJ, 133, 2569

Heald, G., Józsa, G., Serra, P., et al. 2011, A\&A, 526, A118

Helou, G., Soifer, B. T., \& Rowan-Robinson, M. 1985, ApJ, 298, L7

Hess, K. M., Pisano, D. J., Wilcots, E. M., \& Chengalur, J. N. 2009, ApJ, 699, 76

Holwerda, B. W., Pirzkal, N., de Blok, W. J. G., et al. 2011, MNRAS, 416, 2437

Hopkins, A. M., McClure-Griffiths, N. M., \& Gaensler, B. M. 2008, ApJ, 628, L13

Hopkins, P. F., Croton, D., Bundy, K., et al. 2010, ApJ, 724, 915

Huchra, J. P., Macri, L. M., Masters, K. L., et al., 2012, ApJS, 199, 26

Kroupa, P. 2002, Science, 295, 82

Lacey, C., \& Cole, S. 1993, MNRAS, 262, 627

Lambas, D. G., Alonso, S., Mesa, V., \& O'Mill, A. L. 2012, A\&A, 539, A45

Le Fèvre, O., Abraham, R., Lilly, S. J., et al. 2000, MNRAS, 311, 565

Lehner, N., Howk, J. C., Tripp, T. M., et al. 2013, ApJ, 770, 138

Leroy, A. K., Walter, F., Brinks, E., et al. 2008, ApJ, 136, 2782

Lin, L., Patton, D. R., Koo, D. C., et al. 2008, ApJ, 681, 232

Longhetti, M., \& Saracco, P. 2009, MNRAS, 394, 774

López-Sanjuan, C., Balcells, M., \& Pérez-González, P. G. 2009, A\&A, 501, 505

Lotz, J. M., Primack, J., \& Madau, P. 2004, AJ, 128, 163

Lotz, J. M., Davis, M., Faber, S. M., et al. 2008, ApJ, 672, 177

Lutz, R. K. 1980, The Comput. J., 23, 262

Martin, A. M., Papastergis, E., Giovanelli, R., et al. 2010, ApJ, 723, 1359

McGaugh, S. S. 2012, AJ, 143, 40

Nilson, P. 1973, Uppsala General Catalogue of Galaxies, Acta Universitatis Upsaliensis, Uppsala Astronomiska Observatoriums Annaler
Noeske, K. G., Faber, S. M., Weiner, B. J., et al. 2007, ApJ, 660, L47 Noordermeer, E., van der Hulst, J. M., Sancisi, R., Swaters, R. A., \& van Albada, T. S. 2005, A\&A, 442, 137

Marinacci, F., Binney, J. J., Fraternali, F., et al. 2010, MNRAS, 404, 1464

Okvirk, P., Pichon, C., \& Teyssier, R. 2008, MNRAS, 390, 1326

Oosterloo, T., Fraternali, F., \& Sancisi, R. 2007, AJ, 134, 1019

Panter, B., Jimenez, R., Heavens, A. F., \& Charlot, S. 2007, MNRAS, 378, 1550

Patton, D. R., Carlberg, R. G., Marzke, R. O., et al. 2000, ApJ, 536, 153

Pisano, D. J., Barnes, D. G., Gibson, B. K., et al. 2007, ApJ, 662, 959

Pisano, D. J., Barnes, D. G., Staveley-Smith, L., et al. 2011, ApJS, 197, 28

Portinari, L., Sommer-Larsen, J., \& Tantalo, R. 2004, MNRAS, 347, 691

Prochaska, J. X., \& Wolfe, A. M. 2009, ApJ, 696, 1543

Putman, M. E., Peek, J. E. G., \& Joung, M. R. 2012, ApJ, 749, 181

Rand, R. J., \& Benjamin, R. A. 2008, ApJ, 676, 991

Roberts, M. S. 1975, in Galaxies and the Universe, ed. A. Sandage (University of Chicago Press), 309

Robotham, A. S. G., Baldry, I. K., Bland-Hawthorn, J., et al. 2012, MNRAS, 424,1448

Sakai, S., Mould, J. R., Hughe, S. M. G., et al. 2000, ApJ, 529, 2, 698

Sancisi, R., Fraternali, F., Oosterloo, T., \& van der Hulst, T. 2008, A\&ARv, 15, 189

Shull, J. M., Jones, J. R., Danforth, C. W., \& Collins, J. A. 2009, ApJ, 699, 754

Shull, J. M., Smith, B. D., \& Danforth, C. W. 2012, ApJ, 759, 15

Stewart, K. R., Bullock, J. S., Wechsler, R. H., \& Maller, A. H. 2009, ApJ, 702 307

Taylor, C. L., Kobulnicky, H. A., \& Skillman, E. D. 1998, AJ, 116, 2746

Tully, R. B., Rizzi, L., Shaya, E. J., et al., 2009, AJ, 138, 323

Tully, R. B., Courtois, H. M., Dolphin, A. E., et al. 2013, AJ, 146, 86

Tumlinson, J., Thom, C., Werk, J. K., et al. 2013, ApJ, 777, 59

van der Hulst, J. M., van Albada, T. S., \& Sancisi, R. 2001, in Gas and Galaxy Evolution, eds. J. E. Hibbard, M. Rupen, \& J. H. van Gorkom, ASP Conf. Ser. (San Francisco: ASP), 240, 451

Verheijen, M. A. W., Oosterloo, T. A., van Cappellen, W. A., et al. 2008, in The Evolution of Galaxies Through the Neutral Hydrogen Window, eds. R. Minchin, \& E. Momjian, AIP Conf. Ser. (New York: Am. Inst. Phys.), 1035, 265

Wakker, B. P., York, D. G., Howk, J. C., et al. 2007, ApJ, 670, L113

Whiting, M. T. 2012, MNRAS, 421, 3242

Zwaan, M. A., Meyer, M. J., Staveley-Smith, L., \& Webster, R. L. 2005, MNRAS, 359, L30 


\section{Appendix A: global properties of the main galaxies}

In this Appendix, we list the main properties of the spiral galaxies selected from the WHISP sample for this work. Columns are as follows:

Column (1) gives the UGC name.

Column (2) provides an alternative common name, such as NGC, DDO, or IC classifications.

Column (3) provides the adopted distance in Mpc. We preferably used the EDD catalogue (Tully et al. 2009), otherwise, we used the following distance sources, in the given order: Cosmicflows-2 (Tully et al. 2013), NED archive, Hubble flow with $H_{0}=70 \mathrm{~km} \mathrm{~s}^{-1} \mathrm{Mpc}^{-1}$ and systemic velocities corrected for Virgo-infall taken from the HyperLEDA catalogue.

Columns (4) and (5) give the radius $R_{25}$, namely the length of the projected semi-major axis of a galaxy at the isophotal level $25 \mathrm{mag} \operatorname{arcsec}^{-2}$ in the $B$ band. $R_{25}$ is taken from the HyperLEDA catalogue. In Col. (4) the radius is listed in arcminutes, in Col. (5) it is converted into kiloparsecs using the distances in Col. (3).

Column (6) provides the inclination angle derived from the axis ratio in B band as listed in the HyperLEDA catalogue.

Column (7) gives the systemic velocity measured in this work as the average midpoint between the velocities at the $20 \%$ and $50 \%$ of the peak flux of the global HI-line profile.

Column (8) gives the HI-line width at the $20 \%$ of the peak flux of the global HI-line profile, as calculated in this work.

Column (9) provides the total HI mass estimated in this work.

Column (10) gives the adopted total baryonic mass $M_{\mathrm{bar}}$, calculated as described in Sect. 2.3.

Column (11) provides the star formation rate calculated from the $60 \mu \mathrm{m}$ and $100 \mu \mathrm{m}$ IRAS fluxes.

Column (12) gives the total gas accretion rate from minor mergers estimated in this work, including detectable and "hidden" accretion. 
E. M. Di Teodoro and F. Fraternali: Gas accretion from minor mergers in local spiral galaxies

Table A.1. Global properties of the main galaxies selected from the WHISP sample.

\begin{tabular}{|c|c|c|c|c|c|c|c|c|c|c|c|}
\hline $\begin{array}{l}\text { UGC name } \\
\text { (1) }\end{array}$ & $\begin{array}{c}\text { Other name } \\
\text { (2) }\end{array}$ & $\begin{array}{c}D \\
\mathrm{Mpc} \\
(3) \\
\end{array}$ & $\begin{array}{c}R_{25} \\
( \\
(4) \\
\end{array}$ & $\begin{array}{l}R_{25} \\
\mathrm{kpc} \\
(5) \\
\end{array}$ & $\begin{array}{c}i \\
\circ \\
(6) \\
\end{array}$ & $\begin{array}{c}v_{\text {sys }} \\
\mathrm{km} \mathrm{s}^{-1} \\
(7) \\
\end{array}$ & $\begin{array}{c}w_{20} \\
\mathrm{~km} \mathrm{~s}^{-1} \\
(8) \\
\end{array}$ & $\begin{array}{c}M_{\mathrm{HI}} \\
10^{9} M_{\odot} \\
(9) \\
\end{array}$ & $\begin{array}{c}M_{\mathrm{bar}} \\
10^{9} M_{\odot} \\
(10) \\
\end{array}$ & $\begin{array}{c}S F R \\
M_{\odot} / \mathrm{yr} \\
(11) \\
\end{array}$ & $\begin{array}{c}\dot{M}_{\mathrm{HI}} \\
M_{\odot} / \mathrm{yr} \\
(12) \\
\end{array}$ \\
\hline UGC 00094 & NGC 0026 & $68.6^{1}$ & 0.56 & 11 & 47 & 4587 & 320 & 9.63 & 53.34 & 1.17 & 0.04 \\
\hline UGC 00232 & - & $65.3^{2}$ & 0.52 & 10 & 51 & 4837 & 275 & 7.61 & 38.40 & 1.95 & 0.10 \\
\hline UGC 00485 & - & $58.9^{1}$ & 1.15 & 20 & 83 & 5246 & 357 & 21.63 & 45.84 & 1.27 & 0.90 \\
\hline UGC 00528 & NGC 0278 & 12.0 & 1.17 & 4 & 20 & 640 & 138 & 1.32 & 15.68 & 1.02 & 0.01 \\
\hline UGC 00624 & NGC 0338 & $78.3^{1}$ & 0.87 & 20 & 68 & 4770 & 560 & 15.61 & 173.95 & 5.14 & 1.09 \\
\hline UGC 00625 & IC 0065 & 28.3 & 1.29 & 11 & 73 & 2628 & 360 & 7.68 & 27.05 & 1.12 & 0.74 \\
\hline UGC 00690 & - & $74.5^{1}$ & 0.85 & 18 & 46 & 5872 & 325 & 9.61 & 56.80 & 0.54 & 0.64 \\
\hline UGC 00731 & - & 12.0 & 0.93 & 3 & 24 & 639 & 143 & 0.88 & $38.54^{4}$ & 0.21 & 0.01 \\
\hline UGC 00798 & IC 1654 & $69.4^{2}$ & 0.50 & 10 & 40 & 4898 & 222 & 3.96 & 43.96 & 0.60 & 0.11 \\
\hline UGC 01013 & NGC 0536 & $62.5^{1}$ & 1.48 & 27 & 69 & 5187 & 525 & 8.26 & 109.87 & 1.25 & 0.28 \\
\hline UGC 01256 & NGC 0672 & 8.3 & 3.54 & 9 & 67 & 431 & 240 & 7.56 & 13.99 & 0.18 & 0.01 \\
\hline UGC 01437 & NGC 0753 & $54.5^{2}$ & 0.69 & 11 & 51 & 4905 & 339 & 11.58 & 83.23 & 4.09 & 1.74 \\
\hline UGC 01550 & NGC 0801 & $52.2^{1}$ & 1.38 & 21 & 78 & 5764 & 470 & 15.86 & 75.68 & 2.33 & 0.17 \\
\hline UGC 01633 & NGC 0818 & $58.1^{1}$ & 1.09 & 18 & 70 & 4258 & 501 & 11.35 & 88.10 & 2.57 & 0.21 \\
\hline UGC 01810 & - & $109.8^{3}$ & 0.87 & 28 & 69 & 7578 & 602 & 31.64 & 210.16 & 2.37 & 0.88 \\
\hline UGC 01856 & - & $41.3^{2}$ & 1.07 & 13 & 81 & 4804 & 270 & 11.37 & 21.89 & 0.23 & 0.07 \\
\hline UGC 01886 & - & $67.4^{2}$ & 0.26 & 5 & 57 & 4854 & 502 & 25.67 & 121.29 & 0.85 & 0.33 \\
\hline UGC 01913 & NGC 0925 & 9.2 & 5.36 & 14 & 58 & 552 & 222 & 3.85 & 12.90 & 0.64 & $<0.01$ \\
\hline UGC 01993 & - & $107.7^{1}$ & 0.89 & 28 & 75 & 8018 & 526 & 13.70 & 95.49 & 1.24 & 1.03 \\
\hline UGC 02045 & NGC 0972 & 21.7 & 1.66 & 10 & 61 & 1525 & 332 & 2.12 & 45.93 & 4.42 & $<0.01$ \\
\hline UGC 02069 & - & $36.6^{1}$ & 0.62 & 7 & 55 & 3780 & 255 & 4.15 & 17.71 & 1.10 & 0.07 \\
\hline UGC 02080 & IC 0239 & 10.0 & 2.13 & 6 & 24 & 902 & 135 & 5.46 & 11.52 & 0.16 & 0.01 \\
\hline UGC 02082 & - & 14.7 & 2.56 & 11 & 79 & 702 & 215 & 1.36 & 4.64 & 0.04 & 0.01 \\
\hline UGC 02141 & NGC 1012 & 24.7 & 1.04 & 8 & 60 & 987 & 233 & 2.20 & 17.53 & 1.36 & 1.33 \\
\hline UGC 02154 & NGC 1023 & 10.2 & 3.71 & 11 & 70 & 695 & 482 & 2.21 & 44.83 & 0.78 & 0.01 \\
\hline UGC 02183 & NGC 1056 & 21.7 & 0.93 & 6 & 61 & 1540 & 290 & 3.65 & 18.80 & 0.98 & 0.01 \\
\hline UGC 02459 & - & 32.4 & 1.17 & 11 & 83 & 2467 & 337 & 12.30 & 31.48 & 0.59 & 1.28 \\
\hline UGC 02487 & NGC 1167 & $72.2^{3}$ & 0.91 & 19 & 41 & 4953 & 468 & 16.65 & 261.23 & 3.21 & 1.36 \\
\hline UGC 02503 & NGC 1169 & 32.4 & 1.66 & 16 & 54 & 2391 & 461 & 9.69 & 95.99 & 1.12 & 0.12 \\
\hline UGC 02800 & - & $18.9^{1}$ & 1.17 & 6 & 60 & 1187 & 217 & 2.01 & 5.05 & 1.52 & 0.25 \\
\hline UGC 02855 & - & $14.4^{1}$ & 1.77 & 7 & 65 & 1196 & 453 & 6.35 & 49.22 & 2.22 & 0.02 \\
\hline UGC 02916 & - & $68.0^{2}$ & 0.66 & 13 & 24 & 4517 & 336 & 23.12 & 94.12 & 2.45 & 1.40 \\
\hline UGC 03013 & NGC 1530 & 25.4 & 0.91 & 7 & 55 & 2459 & 341 & 8.98 & 53.03 & 2.07 & 0.03 \\
\hline UGC 03137 & - & 22.1 & 1.90 & 12 & 78 & 993 & 216 & 4.41 & 9.32 & 0.15 & 0.02 \\
\hline UGC 03205 & - & $47.6^{2}$ & 0.66 & 9 & 66 & 3588 & 436 & 9.21 & 65.30 & 0.95 & 2.18 \\
\hline UGC 03326 & - & $77.6^{1}$ & 1.66 & 37 & 84 & 4060 & 532 & 19.48 & 135.84 & 2.38 & 0.05 \\
\hline UGC 03334 & NGC1961 & $59.5^{3}$ & 2.23 & 39 & 50 & 3935 & 660 & 39.72 & 422.71 & 9.24 & 0.26 \\
\hline UGC 03354 & - & $52.5^{1}$ & 0.83 & 13 & 70 & 3085 & 441 & 8.89 & 68.85 & 3.22 & 0.02 \\
\hline UGC 03382 & - & $67.2^{3}$ & 0.63 & 12 & 21 & 4501 & 205 & 5.74 & 73.75 & 0.76 & 0.34 \\
\hline UGC 03407 & - & $39.3^{2}$ & 0.56 & 6 & 45 & 3602 & 312 & 1.75 & 22.06 & 0.70 & 1.17 \\
\hline UGC 03422 & - & $77.2^{2}$ & 0.91 & 20 & 62 & 4065 & 416 & 11.05 & 73.40 & 1.08 & 1.00 \\
\hline UGC 03546 & NGC 2273 & 17.9 & 1.15 & 6 & 53 & 1836 & 339 & 1.95 & 19.09 & 0.56 & 0.21 \\
\hline UGC 03574 & - & 17.1 & 0.74 & 4 & 30 & 1441 & 150 & 3.21 & 6.70 & 0.35 & 0.02 \\
\hline UGC 03580 & - & 25.9 & 1.07 & 8 & 57 & 1198 & 236 & 3.81 & 12.87 & 0.48 & 0.02 \\
\hline UGC 03642 & - & $67.4^{2}$ & 0.76 & 15 & 41 & 4498 & 410 & 37.21 & 146.52 & 1.99 & 0.89 \\
\hline UGC 03734 & NGC 2344 & 23.0 & 1.02 & 7 & 24 & 972 & 150 & 1.12 & 14.80 & 0.11 & 0.01 \\
\hline UGC 03759 & NGC 2347 & $88.3^{1}$ & 0.83 & 21 & 44 & 4416 & 468 & 22.39 & 200.89 & 5.72 & 0.40 \\
\hline UGC 03993 & - & $66.3^{3}$ & 0.42 & 8 & 24 & 4365 & 175 & 7.13 & 50.87 & 0.91 & 0.04 \\
\hline UGC 04036 & NGC 2441 & $44.7^{1}$ & 1.00 & 13 & 24 & 3469 & 141 & 4.07 & 31.61 & 0.89 & 0.11 \\
\hline UGC 04165 & NGC 2500 & 15.0 & 1.23 & 5 & 25 & 515 & 113 & 0.97 & 6.82 & 0.35 & $<0.01$ \\
\hline UGC 04256 & NGC 2532 & $51.6^{2}$ & 0.83 & 12 & 34 & 5256 & 175 & 6.73 & 56.96 & 3.70 & 0.10 \\
\hline UGC 04273 & NGC 2543 & 26.3 & 1.23 & 9 & 62 & 2473 & 317 & 4.32 & 20.75 & 1.23 & 0.11 \\
\hline UGC 04284 & NGC 2541 & 11.2 & 1.51 & 5 & 59 & 559 & 210 & 4.91 & 8.32 & 0.08 & $<0.01$ \\
\hline UGC 04458 & NGC 2599 & $68.6^{3}$ & 0.77 & 15 & 32 & 4757 & 285 & 12.52 & 128.09 & 1.39 & 1.09 \\
\hline UGC 04605 & NGC 2654 & 22.7 & 2.23 & 15 & 78 & 1354 & 430 & 6.32 & 35.50 & 0.82 & 0.01 \\
\hline UGC 04666 & NGC 2685 & 16.0 & 2.18 & 10 & 58 & 876 & 303 & 1.96 & 17.39 & 0.14 & 0.22 \\
\hline UGC 04806 & NGC 2770 & 25.5 & 1.73 & 13 & 76 & 1945 & 353 & 5.42 & 19.53 & 0.64 & 0.85 \\
\hline UGC 04838 & NGC 2776 & 36.0 & 1.07 & 11 & 65 & 2626 & 202 & 6.24 & 44.41 & 1.53 & 0.03 \\
\hline UGC 04862 & NGC 2782 & 42.1 & 1.62 & 20 & 42 & 2540 & 196 & 4.12 & 67.81 & 4.49 & 0.01 \\
\hline
\end{tabular}

Notes. ${ }^{(1)}$ Distance from Cosmicflows-2 catalogue. ${ }^{(2)}$ Distance from NED catalogue. ${ }^{(3)}$ Distance from Hubble flow with Virgo-infall corrected systemic velocity. ${ }^{(4)}$ Baryonic mass from the baryonic Tully-Fisher relation. 
Table A.1. continued.

\begin{tabular}{|c|c|c|c|c|c|c|c|c|c|c|c|}
\hline $\begin{array}{l}\text { UGC name } \\
\text { (1) }\end{array}$ & $\begin{array}{c}\text { Other name } \\
\text { (2) }\end{array}$ & $\begin{array}{c}D \\
\mathrm{Mpc} \\
(3)\end{array}$ & $\begin{array}{c}R_{25} \\
( \\
(4)\end{array}$ & $\begin{array}{l}R_{25} \\
\mathrm{kpc} \\
(5)\end{array}$ & $\begin{array}{c}i \\
\circ \\
(6) \\
\end{array}$ & $\begin{array}{c}v_{\text {sys }} \\
\mathrm{km} \mathrm{s}^{-1} \\
(7)\end{array}$ & $\begin{array}{c}w_{20} \\
\mathrm{~km} \mathrm{~s}^{-1} \\
(8)\end{array}$ & $\begin{array}{c}M_{\mathrm{HI}} \\
10^{9} M_{\odot} \\
(9)\end{array}$ & $\begin{array}{c}M_{\mathrm{bar}} \\
10^{9} M_{\odot} \\
(10) \\
\end{array}$ & $\begin{array}{c}S F R \\
M_{\odot} / \mathrm{yr} \\
(11)\end{array}$ & $\begin{array}{c}\dot{M}_{\mathrm{HI}} \\
M_{\odot} / \mathrm{yr} \\
(12)\end{array}$ \\
\hline UGC 05060 & NGC 2893 & 24.0 & 0.51 & 4 & 36 & 1700 & 187 & 0.92 & 6.66 & 0.42 & 0.05 \\
\hline UGC 05079 & NGC 2903 & 8.5 & 6.01 & 15 & 63 & 555 & 390 & 3.95 & 39.88 & 0.95 & $<0.01$ \\
\hline UGC 05251 & NGC 3003 & $19.6^{1}$ & 2.39 & 14 & 77 & 1481 & 294 & 8.89 & 20.10 & 0.40 & 0.02 \\
\hline UGC 05253 & NGC 2985 & 16.6 & 1.82 & 9 & 36 & 1324 & 316 & 11.62 & 55.21 & 0.82 & 0.37 \\
\hline UGC 05351 & NGC 3067 & 20.6 & 1.02 & 6 & 71 & 1487 & 281 & 0.91 & 15.67 & 1.17 & 0.01 \\
\hline UGC 05452 & NGC 3118 & 20.6 & 1.04 & 6 & 78 & 1348 & 216 & 3.41 & 5.91 & 0.07 & 0.02 \\
\hline UGC 05459 & - & 25.8 & 1.90 & 14 & 79 & 1108 & 282 & 4.82 & 18.06 & 0.48 & 0.02 \\
\hline UGC 05532 & NGC 3147 & 39.8 & 2.04 & 24 & 29 & 2812 & 390 & 9.50 & 227.06 & 4.95 & 0.13 \\
\hline UGC 05556 & NGC 3187 & 26.4 & 1.12 & 9 & 71 & 1582 & 276 & 1.09 & 5.48 & 0.48 & 0.06 \\
\hline UGC 05557 & NGC 3184 & 13.0 & 3.71 & 14 & 21 & 593 & 146 & 3.95 & 32.47 & 0.20 & 0.01 \\
\hline UGC 05589 & NGC 3206 & 25.8 & 1.15 & 9 & 59 & 1162 & 182 & 2.61 & 6.50 & 0.03 & 0.06 \\
\hline UGC 05685 & NGC 3254 & 21.8 & 1.17 & 7 & 72 & 1359 & 378 & 4.71 & 24.34 & 0.22 & 0.10 \\
\hline UGC 05717 & NGC 3259 & 24.0 & 0.85 & 6 & 58 & 1675 & 242 & 6.34 & 14.71 & 0.43 & 0.05 \\
\hline UGC 05786 & NGC 3310 & 20.0 & 0.95 & 6 & 40 & 989 & 221 & 3.36 & 17.42 & 3.23 & 0.02 \\
\hline UGC 05789 & NGC 3319 & 13.3 & 1.82 & 7 & 61 & 739 & 215 & 3.36 & 6.76 & 0.06 & 0.01 \\
\hline UGC 05840 & NGC 3344 & 10.0 & 3.38 & 10 & 25 & 589 & 175 & 3.01 & 17.40 & 0.25 & $<0.01$ \\
\hline UGC 05906 & NGC 3380 & 26.1 & 0.77 & 6 & 27 & 1600 & 130 & 0.42 & 9.70 & 0.15 & 0.01 \\
\hline UGC 05909 & NGC 3381 & 25.7 & 1.00 & 7 & 26 & 1633 & 146 & 2.12 & 9.07 & 0.34 & 0.02 \\
\hline UGC 05918 & - & 10.0 & 1.23 & 4 & 12 & 338 & 78 & 0.25 & 0.57 & 0.09 & $<0.01$ \\
\hline UGC 05997 & NGC 3403 & 20.2 & 1.38 & 8 & 68 & 1261 & 303 & 4.09 & 12.89 & 0.46 & 0.03 \\
\hline UGC 06024 & NGC 3448 & 24.0 & 1.48 & 10 & 73 & 1369 & 299 & 6.76 & 21.06 & 1.12 & 0.05 \\
\hline UGC 06128 & NGC 3512 & 26.1 & 0.79 & 6 & 29 & 1388 & 187 & 0.98 & 13.03 & 0.35 & 0.01 \\
\hline UGC 06225 & NGC 3556 & 9.6 & 1.99 & 6 & 65 & 698 & 341 & 3.48 & 22.10 & 0.81 & 0.02 \\
\hline UGC 06263 & NGC 3583 & 33.0 & 1.12 & 11 & 56 & 2134 & 346 & 6.65 & 69.34 & 2.59 & 0.07 \\
\hline UGC 06283 & NGC 3600 & 14.4 & 0.93 & 4 & 72 & 713 & 218 & 2.86 & 6.14 & 0.26 & 0.01 \\
\hline UGC 06537 & NGC 3726 & 17.1 & 2.62 & 13 & 47 & 864 & 284 & 5.05 & 35.11 & 0.46 & 0.01 \\
\hline UGC 06621 & NGC 3786 & 40.0 & 0.97 & 11 & 59 & 2745 & 418 & 4.56 & 42.88 & 1.27 & 0.02 \\
\hline UGC 06778 & NGC 3893 & 17.1 & 1.35 & 7 & 58 & 968 & 311 & 4.76 & 31.95 & 1.59 & 0.99 \\
\hline UGC 06786 & NGC 3900 & 22.5 & 1.29 & 8 & 61 & 1801 & 426 & 3.33 & 25.43 & 0.24 & 0.56 \\
\hline UGC 06787 & NGC 3898 & 22.1 & 1.73 & 11 & 54 & 1170 & 446 & 3.96 & 57.99 & 0.96 & 1.12 \\
\hline UGC 06833 & NGC 3930 & 12.6 & 1.35 & 5 & 42 & 918 & 161 & 0.99 & 7.00 & 0.36 & 0.01 \\
\hline UGC 06870 & NGC 3953 & $19.2^{1}$ & 3.09 & 17 & 62 & 1051 & 403 & 2.35 & 72.36 & 0.30 & 0.09 \\
\hline UGC 06884 & NGC 3963 & $49.1^{2}$ & 1.26 & 18 & 27 & 3189 & 131 & 8.21 & 68.56 & 1.76 & 0.09 \\
\hline UGC 06930 & - & 17.1 & 0.71 & 4 & 42 & 778 & 141 & 2.52 & 4.77 & 0.19 & 0.01 \\
\hline UGC 06964 & NGC 4010 & $19.1^{1}$ & 1.55 & 9 & 78 & 905 & 278 & 1.40 & 8.52 & 0.28 & 0.01 \\
\hline UGC 07030 & NGC 4051 & 17.2 & 2.45 & 12 & 40 & 704 & 241 & 1.43 & 33.28 & 0.86 & 0.01 \\
\hline UGC 07081 & NGC 4088 & $14.5^{1}$ & 3.54 & 15 & 68 & 756 & 381 & 4.15 & 32.18 & 1.51 & 0.01 \\
\hline UGC 07095 & NGC 4100 & $20.3^{1}$ & 2.29 & 14 & 74 & 1075 & 402 & 3.02 & 35.53 & 1.21 & 0.03 \\
\hline UGC 07183 & NGC 4157 & 18.0 & 3.08 & 16 & 80 & 771 & 422 & 6.29 & 54.12 & 1.69 & 0.03 \\
\hline UGC 07222 & NGC 4183 & $16.4^{1}$ & 2.13 & 10 & 81 & 931 & 247 & 2.95 & 7.98 & 0.20 & 0.01 \\
\hline UGC 07256 & NGC 4203 & 15.1 & 1.69 & 7 & 65 & 1088 & 270 & 2.34 & 33.99 & 0.10 & $<0.01$ \\
\hline UGC 07321 & - & 6.0 & 2.39 & 4 & 86 & 407 & 210 & 0.34 & 0.72 & 0.08 & 0.01 \\
\hline UGC 07399 & NGC 4288 & 9.2 & 0.85 & 2 & 41 & 535 & 165 & 0.74 & 1.35 & 0.35 & 0.01 \\
\hline UGC 07483 & NGC 4359 & 16.3 & 0.69 & 3 & 53 & 1271 & 199 & 1.13 & 3.14 & 0.21 & 0.01 \\
\hline UGC 07489 & NGC 4369 & 11.2 & 1.00 & 3 & 17 & 1029 & 88 & 0.43 & 4.83 & 0.33 & 0.02 \\
\hline UGC 07506 & NGC 4384 & 36.0 & 0.63 & 7 & 39 & 2532 & 176 & 1.13 & 12.87 & 0.84 & 0.20 \\
\hline UGC 07766 & NGC 4559 & 8.7 & 5.24 & 13 & 63 & 814 & 256 & 5.43 & 16.21 & 0.22 & $<0.01$ \\
\hline UGC 07989 & NGC 4725 & 12.4 & 4.89 & 18 & 45 & 1210 & 398 & 5.02 & 71.56 & 1.06 & 1.03 \\
\hline UGC 08307 & NGC5033 & $19.1^{1}$ & 9.77 & 54 & 65 & 875 & 425 & 10.43 & 88.98 & 1.76 & 1.27 \\
\hline UGC 08403 & NGC 5112 & 18.5 & 1.51 & 8 & 52 & 969 & 215 & 3.12 & 8.53 & 0.35 & 0.01 \\
\hline UGC 08699 & NGC 5289 & 30.9 & 1.17 & 11 & 72 & 2518 & 352 & 2.76 & 18.81 & 0.23 & 0.02 \\
\hline UGC 08709 & NGC 5297 & 30.9 & 1.86 & 17 & 76 & 2405 & 414 & 12.73 & 50.61 & 1.05 & 0.09 \\
\hline UGC 08711 & NGC 5301 & 20.2 & 1.99 & 12 & 78 & 1508 & 336 & 3.56 & 18.33 & 0.81 & 0.04 \\
\hline UGC 08863 & NGC 5377 & 28.0 & 1.82 & 15 & 67 & 1791 & 382 & 2.24 & 47.16 & 0.42 & 0.01 \\
\hline UGC 08900 & NGC 5395 & $52.7^{2}$ & 1.26 & 19 & 62 & 3458 & 565 & 11.21 & 143.67 & 3.53 & 0.25 \\
\hline UGC 09242 & - & 27.9 & 2.08 & 17 & 86 & 1438 & 215 & 3.20 & 6.43 & 0.21 & 0.01 \\
\hline UGC 09366 & NGC 5676 & 38.9 & 1.82 & 21 & 63 & 2121 & 462 & 6.41 & 137.46 & 5.76 & 1.09 \\
\hline UGC 09431 & NGC 5714 & $38.7^{1}$ & 1.41 & 16 & 80 & 2242 & 356 & 7.51 & 29.58 & 0.66 & 0.14 \\
\hline UGC 09644 & - & $97.9^{3}$ & 0.57 & 16 & 20 & 6664 & 136 & 7.12 & 42.84 & 1.28 & 0.11 \\
\hline UGC 09753 & NGC 5879 & 15.5 & 1.90 & 9 & 68 & 771 & 287 & 1.32 & 10.88 & 0.28 & 0.01 \\
\hline UGC 09797 & NGC 5905 & $46.6^{1}$ & 1.62 & 22 & 50 & 3393 & 374 & 22.70 & 73.72 & 2.56 & 1.25 \\
\hline UGC 09858 & - & 32.2 & 1.95 & 18 & 78 & 2615 & 386 & 10.67 & 28.75 & 0.41 & 1.66 \\
\hline UGC 09969 & NGC 5985 & 43.7 & 1.99 & 25 & 60 & 2515 & 542 & 10.76 & 144.58 & 1.19 & 0.14 \\
\hline
\end{tabular}


E. M. Di Teodoro and F. Fraternali: Gas accretion from minor mergers in local spiral galaxies

Table A.1. continued.

\begin{tabular}{|c|c|c|c|c|c|c|c|c|c|c|c|}
\hline $\begin{array}{l}\text { UGC name } \\
\text { (1) }\end{array}$ & $\begin{array}{c}\text { Other name } \\
\text { (2) }\end{array}$ & $\begin{array}{c}D \\
\mathrm{Mpc} \\
(3)\end{array}$ & $\begin{array}{c}R_{25} \\
( \\
(4)\end{array}$ & $\begin{array}{l}R_{25} \\
\mathrm{kpc} \\
(5)\end{array}$ & $\begin{array}{c}i \\
\circ \\
(6) \\
\end{array}$ & $\begin{array}{c}v_{\text {sys }} \\
\mathrm{km} \mathrm{s}^{-1} \\
(7)\end{array}$ & $\begin{array}{c}w_{20} \\
\mathrm{~km} \mathrm{~s}^{-1} \\
(8)\end{array}$ & $\begin{array}{c}M_{\mathrm{HI}} \\
10^{9} M_{\odot} \\
(9)\end{array}$ & $\begin{array}{c}M_{\mathrm{bar}} \\
10^{9} M_{\odot} \\
(10)\end{array}$ & $\begin{array}{c}S F R \\
M_{\odot} / \mathrm{yr} \\
(11) \\
\end{array}$ & $\begin{array}{c}\dot{M}_{\mathrm{HI}} \\
M_{\odot} / \mathrm{yr} \\
(12)\end{array}$ \\
\hline UGC 10359 & NGC 6140 & 16.0 & 1.04 & 5 & 44 & 908 & 221 & 5.41 & 11.61 & 0.14 & 0.01 \\
\hline UGC 10445 & - & 18.1 & 0.95 & 5 & 45 & 962 & 159 & 2.23 & 6.94 & 0.16 & 0.05 \\
\hline UGC 10448 & NGC 6186 & $154.0^{2}$ & 0.79 & 35 & 41 & 11352 & 118 & 9.56 & 439.96 & 8.82 & 0.02 \\
\hline UGC 10470 & NGC 6217 & 23.0 & 1.12 & 7 & 34 & 1355 & 192 & 5.94 & 30.66 & 1.86 & 0.02 \\
\hline UGC 10497 & - & $65.6^{2}$ & 0.59 & 11 & 65 & 4296 & 267 & 8.93 & 21.34 & 0.36 & 0.17 \\
\hline UGC 10564 & NGC 6237 & 21.0 & 0.62 & 4 & 52 & 1129 & 175 & 5.64 & 11.08 & 0.27 & 0.03 \\
\hline UGC 11124 & - & 25.0 & 1.12 & 8 & 26 & 1599 & 153 & 2.23 & 11.71 & 0.16 & 0.03 \\
\hline UGC 11218 & NGC 6643 & 20.6 & 1.66 & 10 & 61 & 1484 & 350 & 3.20 & 30.27 & 1.78 & 0.04 \\
\hline UGC 11269 & NGC 6667 & 44.9 & 0.93 & 12 & 56 & 2581 & 412 & 13.36 & 66.90 & 1.73 & 0.02 \\
\hline UGC 11283 & IC 1291 & 30.0 & 0.66 & 6 & 35 & 1946 & 198 & 2.55 & 9.44 & 0.38 & 0.55 \\
\hline UGC 11429 & NGC 6792 & $62.2^{1}$ & 1.04 & 19 & 58 & 4637 & 510 & 12.26 & 129.81 & 1.60 & 0.31 \\
\hline UGC 11466 & - & 18.1 & 0.74 & 4 & 53 & 821 & 237 & 2.79 & 10.96 & 0.84 & 0.01 \\
\hline UGC 11670 & NGC 7013 & 15.0 & 2.08 & 9 & 71 & 775 & 340 & 1.35 & 26.16 & 0.28 & $<0.01$ \\
\hline UGC 11852 & - & $82.4^{2}$ & 0.46 & 11 & 44 & 5845 & 328 & 26.73 & 82.67 & 0.96 & 0.45 \\
\hline UGC 11861 & - & 14.4 & 0.89 & 4 & 61 & 1482 & 259 & 2.10 & 10.25 & 0.47 & 0.02 \\
\hline UGC 11909 & - & 14.1 & 1.00 & 4 & 78 & 1105 & 242 & 2.87 & 7.78 & 0.39 & 0.01 \\
\hline UGC 11914 & NGC 7217 & 15.0 & 2.29 & 10 & 35 & 950 & 301 & 0.70 & 52.88 & 0.68 & $<0.01$ \\
\hline UGC 11951 & NGC 7231 & 14.2 & 0.85 & 4 & 69 & 1086 & 223 & 1.56 & 4.97 & 0.35 & 0.92 \\
\hline UGC 11994 & - & $65.8^{1}$ & 1.04 & 20 & 82 & 4882 & 436 & 6.95 & 57.44 & 1.69 & 0.29 \\
\hline UGC 12554 & NGC 7640 & 9.9 & 4.06 & 12 & 78 & 363 & 238 & 3.05 & 8.78 & 0.24 & $<0.01$ \\
\hline UGC 12693 & - & $60.5^{1}$ & 0.55 & 10 & 78 & 4958 & 236 & 9.67 & 15.50 & 0.69 & 0.12 \\
\hline UGC 12732 & - & 15.1 & 1.38 & 6 & 28 & 728 & 131 & 1.96 & 4.06 & 0.59 & 0.01 \\
\hline UGC 12754 & NGC 7741 & $13.6^{1}$ & 1.82 & 7 & 49 & 749 & 202 & 1.78 & 5.76 & 0.36 & 0.01 \\
\hline UGC 12808 & NGC 7769 & $61.5^{3}$ & 0.87 & 16 & 68 & 4225 & 326 & 4.79 & 134.32 & 6.21 & 0.05 \\
\hline
\end{tabular}

